\title{
DYNAMICS OF COMPLEX-VALUED MODIFIED KDV SOLITONS WITH APPLICATIONS TO THE STABILITY OF BREATHERS
}

\author{
MigUEL A. ALEJO AND CLAUDIO MUÑOZ
}

\begin{abstract}
We study the long-time dynamics of complex-valued modified Korteweg-de Vries (mKdV) solitons, which are recognized because they blowup in finite time. We establish stability properties at the $H^{1}$ level of regularity, uniformly away from each blow-up point. These new properties are used to prove that $\mathrm{mKdV}$ breathers are $H^{1}$ stable, improving our previous result [4, where we only proved $H^{2}$ stability. The main new ingredient of the proof is the use of a Bäcklund transformation which relates the behavior of breathers, complex-valued solitons and small real-valued solutions of the mKdV equation. We also prove that negative energy breathers are asymptotically stable. Since we do not use any method relying on the Inverse Scattering Transform, our proof works even under $L^{2}(\mathbb{R})$ perturbations, provided a corresponding local well-posedness theory is available.
\end{abstract}

\section{Contents}

1. Introduction

2. Complex-valued $\mathrm{mKdV}$ soliton profiles

3. Bäcklund transformation for $\mathrm{mKdV}$

4. Dynamics of complex-valued $\mathrm{mKdV}$ solitons

5. Complex solitons versus breathers 24

6. Double Bäcklund transformation for $\mathrm{mKdV}$

7. Stability of breathers 36

8. Asymptotic Stability 40

Appendix A. Proof of Lemma 5.1 42

References

\section{INTRODUCTION}

Consider the modified Korteweg-de Vries $(\mathrm{mKdV})$ equation on the real line

$$
u_{t}+\left(u_{x x}+u^{3}\right)_{x}=0
$$

where $u=u(t, x)$ is a complex-valued function, and $(t, x) \in \mathbb{R}^{2}$. Note that (1.1) is not $U(1)$-invariant. In the case of real-valued initial data, the associated Cauchy problem for (1.1) is globally well-posed for initial data in $H^{s}(\mathbb{R})$, for any $s>\frac{1}{4}$,

Date: March 1, 2022.

2000 Mathematics Subject Classification. Primary 35Q51, 35Q53; Secondary 37K10, 37K40.

Key words and phrases. mKdV equation, Bäcklund transformation, solitons, breather, stability. 
see Kenig-Ponce-Vega 23, and Colliander, Keel, Staffilani, Takaoka and Tao 13 . Additionally, the (real-valued) flow map is not uniformly continuous if $s<\frac{1}{4}[24$ ] 1 ] In order to prove this last result, Kenig, Ponce and Vega considered a very particular class of solutions of (1.1) called breathers, discovered by Wadati in [38.

Definition 1.1 (See e.g. 38, 25). Let $\alpha, \beta>0$ and $x_{1}, x_{2} \in \mathbb{R}$ be fixed parameters. The $m K d V$ breather is a smooth solution of (1.1) given explicitly by the formula

$$
B:=B\left(t, x ; \alpha, \beta, x_{1}, x_{2}\right):=2 \sqrt{2} \partial_{x}\left[\arctan \left(\frac{\beta}{\alpha} \frac{\sin \left(\alpha y_{1}\right)}{\cosh \left(\beta y_{2}\right)}\right)\right],
$$

where

$$
y_{1}:=x+\delta t+x_{1}, \quad y_{2}:=x+\gamma t+x_{2},
$$

and

$$
\delta:=\alpha^{2}-3 \beta^{2}, \quad \gamma:=3 \alpha^{2}-\beta^{2} .
$$

Breathers are oscillatory bound states. They are periodic in time (after a suitable space shift) and localized in space. The parameters $\alpha$ and $\beta$ are scaling parameters, $x_{1}, x_{2}$ are shifts, and $-\gamma$ represents the velocity of a breather. As we will see later, the main difference between soliton $\mathbf{2}^{2}$ and breathers is given at the level of the oscillatory scaling $\alpha$, which is not present in the case of solitons. For a detailed account of the physics of breathers see e.g. 25, 1, 6, 2, 4, and references therein.

Numerical computations (see Gorria-Alejo-Vega 3]) showed that breathers are numerically stable. Next, in [4 we constructed a Lyapunov functional that controls the dynamics of $H^{2}$-perturbations of (1.2). The purpose of this paper is to improve our previous result [4] and show that $\mathrm{mKdV}$ breathers are indeed $H^{1}$ stable, i.e. stable in the energy space.

Theorem 1.2. Let $\alpha, \beta>0$ be fixed scalings. There exist parameters $\eta_{0}, A_{0}$, depending on $\alpha$ and $\beta$ only, such that the following holds. Consider $u_{0} \in H^{1}(\mathbb{R})$, and assume that there exists $\eta \in\left(0, \eta_{0}\right)$ such that

$$
\left\|u_{0}-B(0, \cdot ; \alpha, \beta, 0,0)\right\|_{H^{1}(\mathbb{R})} \leq \eta .
$$

Then there exist functions $x_{1}(t), x_{2}(t) \in \mathbb{R}$ such that the solution $u(t)$ of the Cauchy problem for the $m K d V$ equation (1.1), with initial data $u_{0}$, satisfies

$$
\begin{gathered}
\sup _{t \in \mathbb{R}}\left\|u(t)-B\left(t, \cdot ; \alpha, \beta, x_{1}(t), x_{2}(t)\right)\right\|_{H^{1}(\mathbb{R})} \leq A_{0} \eta, \\
\sup _{t \in \mathbb{R}}\left|x_{1}^{\prime}(t)\right|+\left|x_{2}^{\prime}(t)\right| \leq C A_{0} \eta,
\end{gathered}
$$

for some constant $C>0$.

The initial condition (1.5) can be replaced by any initial breather profile of the form $B\left(t_{0} ; \alpha, \beta, x_{1}^{0}, x_{2}^{0}\right)$, with $t_{0}, x_{1}^{0}, x_{2}^{0} \in \mathbb{R}$, thanks to the invariance of the equation under translations in time and space 3 Moreover, using the Miura transform [17, one can prove a natural stability property in $L^{2}(\mathbb{R} ; \mathbb{C})$ for the associated complexvalued $\mathrm{KdV}$ breather.

\footnotetext{
${ }^{1}$ However, one can construct a solution in $L^{2}$, see 12 .

${ }^{2}$ See (1.8).

${ }^{3}$ Indeed, if $u(t, x)$ solves (1.1), then for any $t_{0}, x_{0} \in \mathbb{R}$, and $c>0, u\left(t-t_{0}, x-x_{0}\right)$, $c^{1 / 2} u\left(c^{3 / 2} t, c^{1 / 2} x\right), u(-t,-x)$ and $-u(t, x)$ are solutions of 1.1.).
} 
Additionally, from the proof the shifts $x_{1}(t)$ and $x_{2}(t)$ in Theorem 1.2 can be described almost explicitly 4 , which is a sustainable improvement with respect to our previous result 4, where no exact control on the shift parameters was given. We recall that we obtain such a control with no additional decay assumptions on the initial data other than being in $H^{1}(\mathbb{R})$.

Theorem 1.2 places breathers as stable objects at the same level of regularity as $\mathrm{mKdV}$ solitons, even if they are very different in nature. To be more precise, a (real-valued) soliton is a solution of (1.1) of the form

$$
u(t, x)=Q_{c}(x-c t), \quad Q_{c}(s):=\sqrt{c} Q(\sqrt{c} s), \quad c>0,
$$

with

$$
Q(s):=\frac{\sqrt{2}}{\cosh (s)}=2 \sqrt{2} \partial_{s}\left[\arctan \left(e^{s}\right)\right],
$$

and where $Q_{c}>0$ satisfies the nonlinear ODE

$$
Q_{c}^{\prime \prime}-c Q_{c}+Q_{c}^{3}=0, \quad Q_{c} \in H^{1}(\mathbb{R}) .
$$

We recall that solitons are $H^{1}$-stable (Benjamin [7, Bona-Souganidis-Strauss [9]). See also the works by Grillakis-Shatah-Strauss 18 and Weinstein 40 for the nonlinear Schrödinger case.

Even more surprising is the fact that Theorem 1.2 will arise as a consequence of a suitable stability property of the zero solution and of complex-valued $\mathrm{mKdV}$ solitons, which are singular solutions.

A complex-valued soliton is a solution of the form (1.8) of (1.1), with a complexvalued scaling and velocity, i.e.,

$$
u(t, x):=Q_{c}(x-c t), \quad \sqrt{c}:=\beta+i \alpha, \quad \alpha, \beta>0,
$$

see Definition 2.1 for a rigorous interpretation. In Lemma 2.2 we give a detailed description of the singular nature of (1.10). On the other hand, very little is known about $\mathrm{mKdV}$ (1.1) when the initial data is complex-valued. For instance, it is known that it has finite time blow-up solutions, the most important examples being the complex solitons themselves, see e.g. Bona-Vento-Weissler [10] and references therein for more details. According to [10, blow-up in the complex-valued case can be understood as the intersection with the real line $x \in \mathbb{R}$ of a curve of poles of the solution after being extended to the complex plane (i.e., now $x$ is replaced by $z \in \mathbb{C}$ ). Blow-up in this case seems to have better properties than the corresponding critical blow-up described by Martel and Merle in [30.

Let $H^{1}(\mathbb{R} ; \mathbb{C})$ denote the standard Sobolev space of complex-valued functions $f(x) \in \mathbb{C}, x \in \mathbb{R}$. In this paper we prove the following stability property for solitons, far away from each blow-up time.

Theorem 1.3. There exists an open set of initial data in $H^{1}(\mathbb{R} ; \mathbb{C})$ for which the $m K d V$ complex solitons are well-defined and stable in $H^{1}(\mathbb{R} ; \mathbb{C})$ for all times uniformly separated from a countable sequence of finite blow-up times with no limit points. Moreover, one can define a mass and an energy, both invariant for all time.

\footnotetext{
${ }^{4}$ See equation 7.6 .
} 
We cannot prove an all-time stability result using the $H^{1}(\mathbb{R} ; \mathbb{C})$-norm because even complex solitons leave that space at each blow-up time, and several computations in this paper break down. However, the previous result states that the Cauchy problem is almost globally well-posed around a soliton, and the solution can be continued after (or before) every blow-up time. The novelty with respect to the local Cauchy theory [23] is that now it is possible to define an almost global solution instead of defining a local solution on each subinterval of time defined by two blow-up points, because from the proof we will recognize that the behavior before and after the blow-up time are deeply linked. From this property the existence and invariance of uniquely well-defined mass and energy will be quite natural. For this particular problem, we answer positively the questions about existence, uniqueness and regularity after blow-up posed by Merle in 33 . See Theorem 4.5 and its corollaries for a more detailed statement.

We finally prove that breathers behaving as standard solitons are asymptotically stable in the energy space. For previous results for the soliton and multi-soliton case, see Pego-Weinstein [35] and Martel-Merle 31.

Theorem 1.4. Under the hypotheses of Theorem 1.2, there exists $c_{0}>0$ depending on $\eta$, with $c_{0}(\eta) \rightarrow 0$ as $\eta \rightarrow 0$, such that the following holds. There exist $\beta^{*}$ and $\alpha^{*}$ close enough to $\beta$ and $\alpha$ respectively (depending on $\eta$ ), for which

$$
\lim _{t \rightarrow+\infty}\left\|u(t)-B\left(t ; \cdot, \alpha^{*}, \beta^{*}, x_{1}(t), x_{2}(t)\right)\right\|_{H^{1}\left(x \geq c_{0} t\right)}=0 .
$$

In particular, the asymptotic of the solution $u(t)$ has new and explicit velocity parameters $\delta^{*}=\left(\alpha^{*}\right)^{2}-3\left(\beta^{*}\right)^{2}$ and $\gamma^{*}=3\left(\alpha^{*}\right)^{2}-\left(\beta^{*}\right)^{2}$ at the main order.

The previous result is more interesting when $\gamma<0$, see (1.4). In this case, the breather has negative energy (see [4, p. 9]), and it moves rightwards in space (the so-called physically relevant region). Theorem 1.4 states that breathers almost clean the right portion of the real line. We recall that working in the energy space implies that small solitons moving to the right in a very slow fashion are allowed (the condition $c_{0}>0$ is essential, see e.g. Martel-Merle 31]). Indeed, there are explicit solutions of (1.1) composed by one breather and one very small soliton moving rightwards, that contradicts any sort of global asymptotic stability result in the energy space 25. Additionally, we cannot ensure that the left portion of the real line $\{x<0\}$ corresponds to radiation only. Following [25, it is possible to construct a solution to (1.1) composed by two breathers, one very small with respect to the other one, the latter with positive velocity, and the former with small but still negative velocity (just take the corresponding scaling parameters $\alpha$ and $\beta$ both small such that $-\gamma<0$ ). Such a solution has no radiation at infinity. Of course, working in a neighborhood of the breather using weighted spaces rules out such small perturbations.

The mechanism under which $\alpha^{*}$ and $\beta^{*}$ are chosen is very natural and reflects the power and simplicity of the arguments of the proof: under different scaling parameters, it was impossible to describe the dynamics as in Theorem 1.2. We are indeed under two linked results: in some sense Theorem 1.2 is a consequence of Theorem 1.4 and vice versa.

On the other hand, the fact that no shifts in (1.11) are needed can be contrasted with the Martel-Merle computations in 29. In that paper they calculated the 
leading order of the shift perturbation for the small-large soliton collision in the $\mathrm{mKdV}$ case. It was found that such shifts are very small $\left(\sim \eta^{2}\right)$ compared with the size of the perturbation $(\sim \eta)$.

Finally, concerning the portion of the mass not considered in (1.11), we have the following characterization of inelasticity.

Corollary 1.5. Assume that $u_{0}$ in (1.5) is non trivial, i.e.,

$$
\ell_{0}:=\left\|u_{0}-B(0, \cdot ; \alpha, \beta, 0,0)\right\|_{H^{1}(\mathbb{R})}>0 .
$$

Then there exists $c_{0}>0$ independent of $\eta$ such that

$$
\liminf _{t \rightarrow+\infty}\left\|u(t)-B\left(t ; \cdot, \alpha^{*}, \beta^{*}, 0,0\right)\right\|_{H^{1}(\mathbb{R})} \geq c_{0} \ell_{0} .
$$

Moreover, we have

$$
c_{0} \ell_{0} \leq\left|\beta^{*}-\beta\right|+\left|\alpha^{*}-\alpha\right| \leq \frac{1}{c_{0}} \ell_{0} .
$$

It is also important to emphasize that (1.1) is a well-known completely integrable model [17, 1, 25, 26, 36, with infinitely many conserved quantities, and a suitable Lax-pair formulation. The Inverse Scattering Theory has been applied in [36] to describe the evolution of rapidly decaying initial data, by purely algebraic methods. Solutions are shown to decompose into a very particular set of solutions: solitons, breathers and radiation. Moreover, as a consequence of the integrability property, these nonlinear modes interact elastically during the dynamics, and no dispersive effects are present at infinity. In particular, even more complex solutions are present, such as multi-solitons (explicit solutions describing the interaction of several solitons [20]). Multi-solitons for $\mathrm{mKdV}$ and several integrable models of Korteweg-de Vries type are stable in $H^{1}$, see Maddocks-Sachs [27] for the KdV case and in a more general setting see Martel-Merle-Tsai [32.

However, the proof of Theorem 1.2 does not involve any method relying on the Inverse Scattering transform [17, 36], nor the steepest descent machinery [15, 5 which allows to work in the very large energy space $H^{1}(\mathbb{R})$. Note that if the Inverse Scattering methods are allowed, one could describe the dynamics of very general initial data with more detail. But if this is the case, additional decay and/or spectral assumptions are always needed, and except by well-prepared initial data, such conditions are difficult to verify. We claim that our proof works even if the initial data is in $L^{2}(\mathbb{R})$, provided $m K d V$ is locally well-posed at that level of regularity, which remains a very difficult open problem.

Comparing with [4, where we have proved that mKdV breathers are $H^{2}$-stable, now we are not allowed to use the third conservation law associated to mKdV 6

$$
F[u](t)=\frac{1}{2} \int_{\mathbb{R}} u_{x x}^{2}(t, x) d x-\frac{5}{2} \int_{\mathbb{R}} u^{2} u_{x}^{2}(t, x) d x+\frac{1}{4} \int_{\mathbb{R}} u^{6}(t, x) d x,
$$

nor the elliptic equation satisfied by any breather profile:

$$
B_{(4 x)}-2\left(\beta^{2}-\alpha^{2}\right)\left(B_{x x}+B^{3}\right)+\left(\alpha^{2}+\beta^{2}\right)^{2} B+5 B B_{x}^{2}+5 B^{2} B_{x x}+\frac{3}{2} B^{5}=0,
$$

\footnotetext{
${ }^{5}$ Note that in [15] the authors consider the defocusing mKdV equation, which has no smooth solitons and breathers.

${ }^{6}$ See 4.13) and 4.14 for the other two low-regularity conserved quantities.
} 
since the dynamics is no longer in $H^{2}$. Moreover, since breathers are bound states, there is no associated decoupling in the dynamics as time evolves as in the MartelMerle-Tsai paper [32, which makes the proof of the $H^{1}$ case even more difficult. We need a different method of proof.

In this paper we follow a method of proof that it is in the spirit of the seminal work by Merle and Vega 34 (see also Alejo-Muñoz-Vega [5), where the $L^{2}$-stability of KdV solitons has been proved. In those cases the use of the Miura and Gardner transformations were the new ingredients to prove stability where the standard energy is missing. Recently, the Miura transformation has been studied at very low regularity. Using this information, Buckmaster and Koch showed that KdV solitons are stable even under $H^{-1} \cap H^{-3 / 4}$ perturbations [11.

More precisely, in this paper we will make use of the Bäcklund transformation [25. p. 257] associated to $\mathrm{mKdV}$ to obtain new conserved quantities, additional to the mass and energy. We point out the recent works by Mizumachi-Pelinovsky [28] and Hoffmann-Wayne 21], where a similar approach was described for the NLS and sine-Gordon equations and their corresponding one-solitons. However, unlike those previous works, and in order to control any breather, we use the Bäcklund transformation twice: one to control an associated complex-valued mKdV soliton, and a second one to get almost complete control of the breather.

Indeed, solving the Bäcklund transformation in a vicinity of a breather leads (formally) to the emergence of complex-valued $\mathrm{mKdV}$ solitons, which blow-up in finite time. A difficult problem arises at the level of the Cauchy theory, and any attempt to prove stability must face the ill-posedness behavior of the complex-valued $\mathrm{mKdV}$ equation (1.1). However, after a new use of the Bäcklund transformation around the complex soliton we end-up with a small, real-valued $H^{1}(\mathbb{R})$ solution of $\mathrm{mKdV}$, which is stable for all time. The fact that a second application of the Bäcklund transformation leads to a real-valued solution is not trivial and is a consequence of a deep property called permutability theorem [25]. Roughly speaking, that result states that the order under which we perform two inversions of the Bäcklund transformation does not matter. After some work we are able to give a rigorous proof of the following fact: we can invert a breather using Bäcklund towards two particularly well chosen complex solitons first, and then invert once again to obtain two small solutions -say $a$ and $b$-, and the final result must be the same. Even better, one can show that $a$ has to be the conjugate of $b$, which gives the real character of the solution. Now the dynamics is real-valued and simple. We use the Kenig-Ponce-Vega 23 theory to evolve the system to any given time. Using this trick we avoid dealing with the blow-up times of the complex soliton -for a whileand at the same time we prove a new stability result for them.

However, unlike the previous results [28, 21, we cannot invert the Bäcklund transformation at any given time, and in fact each blow-up time of the complexvalued $\mathrm{mKdV}$ soliton is a dangerous obstacle for the breather stability. In order to extend the stability property up to the blow-up times we discard the method involving the Bäcklund transformation. Instead we run a bootstrap argument starting from a fixed time very close to each singular point, using the fact the real-valued $\mathrm{mKdV}$ dynamics is continuous in time. Finally, using energy methods related to the stability of single solitons we are able to extend the uniform bounds in time to any singularity point, with a universal constant $A_{0}$ as in Theorem 1.2 . 
From the proof it will be evident that even if there is no global well-posedness theory (with uniform bounds in time) below $H^{s}, s<\frac{1}{4}$, one can prove stability of breathers in spaces of the form $H^{1} \cap H^{s}, s<\frac{1}{4}$, following the ideas of Buckmaster and Koch [11. We thank professor Herbert Koch for mentioning to us this interesting property.

Our results apply without important modifications to the case of the sine-Gordon (SG) equation in $\mathbb{R}_{t} \times \mathbb{R}_{x}$

$$
u_{t t}-u_{x x}+\sin u=0, \quad\left(u, u_{t}\right)(t, x) \in \mathbb{R}^{2},
$$

and its corresponding breather [25, p. 149]. See [8, 14, 37, for related results. Note that SG is globally well-posed in $L^{2} \times H^{-1}$; then we have that breathers are stable under small perturbations in that space. Since the proofs are very similar, and in order to make this paper non redundant, we skip the details.

Moreover, following our proof it is possible to give a new proof of the global $H^{1}$-stability of two-solitons proved by Martel, Merle and Tsai in [32.

We also claim that $k$-breathers $(k \geq 2)$, namely solutions composed by $k$ different breathers are also $H^{1}$-stable. Following the proof of Theorem [1.2 one can show by induction that a $k$-breather can be obtained from a $(k-1)$-breather after two Bäcklund transformations using a fixed set of complex conjugate parameters, as in Lemmas 2.4 and 5.1. After proving this identity, the rest of the proof adapts with no deep modifications.

This paper is organized as follows. In Section 2 we introduce the complexvalued soliton profiles. Section 3 is devoted to the study of the mKdV Bäcklund transformation in the vicinity of a given complex-valued mKdV solution. In Section

4 we apply the previous results to prove Theorem 1.3 (see Theorem 4.5). Section 5 deals with the relation between complex soliton profiles and breathers. In Section 6 we apply the results from Section 3 to the case of a perturbation of a breather solution. Finally, in Sections 7 and 8 we prove Theorems 1.2 and 1.4 and Corollary 1.5 .

Acknowledgments. The first author acknowledges the financial support from the University of the Basque Country-EHU. Part of this work was done while the second author was L.E. Dickson Instructor at the University of Chicago. He would like to express his gratitude with the members of the Department of Mathematics, in particular professors Carlos Kenig and Wilhelm Schlag. We also thank Herbert Koch and Yvan Martel for several enlightening discussions which improved the quality of this paper, and a gap in the first version of this one.

\section{CompleX-Valued mKdV SOliton PROFiles}

First of all, we recall the well-known complex-valued mKdV profile.

Definition 2.1. Consider parameters $\alpha, \beta>0, x_{1}$ and $x_{2} \in \mathbb{R}$. We introduce the complex-valued kink profile

$$
\widetilde{Q}=\widetilde{Q}\left(x ; \alpha, \beta, x_{1}, x_{2}\right),
$$

defined as

$$
\widetilde{Q}:=2 \sqrt{2} \arctan \left(e^{\beta y_{2}+i \alpha y_{1}}\right),
$$


where $y_{1}$ and $y_{2}$ are (re) defined as

$$
y_{1}:=x+x_{1}, \quad y_{2}:=x+x_{2} .
$$

Note that

$$
\widetilde{Q}(-\infty)=0, \quad \widetilde{Q}(+\infty)=\sqrt{2} \pi .
$$

We define the complex-valued soliton profile as follows:

$$
\begin{aligned}
Q:= & \partial_{x} \widetilde{Q} \\
= & \frac{2 \sqrt{2}(\beta+i \alpha) e^{\beta y_{2}+i \alpha y_{1}}}{1+e^{2\left(\beta y_{2}+i \alpha y_{1}\right)}} \\
= & \sqrt{2} \frac{\beta \cosh \left(\beta y_{2}\right) \cos \left(\alpha y_{1}\right)+\alpha \sinh \left(\beta y_{2}\right) \sin \left(\alpha y_{1}\right)}{\cosh ^{2}\left(\beta y_{2}\right)-\sin ^{2}\left(\alpha y_{1}\right)}+ \\
& +i \sqrt{2} \frac{\alpha \cosh \left(\beta y_{2}\right) \cos \left(\alpha y_{1}\right)-\beta \sinh \left(\beta y_{2}\right) \sin \left(\alpha y_{1}\right)}{\cosh ^{2}\left(\beta y_{2}\right)-\sin ^{2}\left(\alpha y_{1}\right)} .
\end{aligned}
$$

Finally we denote

$$
\widetilde{Q}_{t}:=-(\beta+i \alpha)^{2} Q
$$

and

$$
\widetilde{Q}_{1}:=\partial_{x_{1}} \widetilde{Q}, \quad \widetilde{Q}_{2}:=\partial_{x_{2}} \widetilde{Q} .
$$

Note that $Q$ is complex-valued and is pointwise convergent to the soliton $Q_{\beta^{2}}$ as $\alpha \rightarrow 0$. A second condition satisfied by $\widetilde{Q}$ and $Q$ is the following periodicity property: for all $k \in \mathbb{Z}$,

$$
\left\{\begin{array}{l}
\widetilde{Q}\left(x ; \alpha, \beta, x_{1}+\frac{k \pi}{\alpha}, x_{2}\right)=(-1)^{k} \widetilde{Q}\left(x ; \alpha, \beta, x_{1}, x_{2}\right), \\
Q\left(x ; \alpha, \beta, x_{1}+\frac{k \pi}{\alpha}, x_{2}\right)=(-1)^{k} Q\left(x ; \alpha, \beta, x_{1}, x_{2}\right) .
\end{array}\right.
$$

In what follows, we remark that $\widetilde{Q}$ and $Q$ may blow-up in finite time.

Lemma 2.2. Consider the complex-valued soliton profile defined in (2.1)-(2.5). Assume that

$$
\text { for } x_{2} \text { fixed and some } k \in \mathbb{Z}, \quad x_{1}=x_{2}+\frac{\pi}{\alpha}\left(k+\frac{1}{2}\right) .
$$

Then $\widetilde{Q}$ and $Q$ cannot be defined at $x=-x_{2}$. Moreover, if $x_{1}=x_{2}=0$, we have $Q(\cdot ; \alpha, \beta, 0,0) \in H^{1}(\mathbb{R} ; \mathbb{C})$.

Remark 2.1. We emphasize that, given $x_{2}$ fixed, the set of points $x_{1}$ of the form (2.9) for some $k \in \mathbb{Z}$ is a countable set of real numbers with no limit points.

Proof. Fix $x_{2} \in \mathbb{R}$. If (2.9) is satisfied for some $k \in \mathbb{Z}$, we have that at $x=-x_{2}$,

$$
y_{1}=x+x_{1}=\frac{\pi}{\alpha}\left(k+\frac{1}{2}\right), \quad y_{2}=x+x_{2}=0,
$$

and

$$
\sinh \left(\beta y_{2}\right)=0, \quad \cos \left(\alpha y_{1}\right)=0
$$

Therefore, under (2.9), we have from (2.1) and (2.5) that $\widetilde{Q}$ and $Q$ cannot be defined at $x=-x_{2}$. Finally, if $x_{1}=x_{2}=0$, we have

$$
k+\frac{1}{2}=0, \quad k \in \mathbb{Z},
$$


which is impossible.

Lemma 2.3. Fix $\alpha, \beta>0$ and $x_{1}, x_{2} \in \mathbb{R}$ such that (2.9) is not satisfied. Then we have

$$
Q_{x x}-(\beta+i \alpha)^{2} Q+Q^{3}=0, \quad \text { for all } x \in \mathbb{R},
$$

and

$$
Q_{x}^{2}-(\beta+i \alpha)^{2} Q^{2}+\frac{1}{2} Q^{4}=0, \quad \text { for all } x \in \mathbb{R} .
$$

Moreover, the previous identities can be extended to any $x_{1}, x_{2} \in \mathbb{R}$ by continuity.

Proof. Direct from the definition.

Assume that (2.9) does not hold. Consider the sin and cos functions applied to complex numbers. We have from (2.1) and (2.4),

$$
\begin{aligned}
\sin \left(\frac{\widetilde{Q}}{\sqrt{2}}\right) & =\sin \left(2 \arctan e^{\beta y_{2}+i \alpha y_{1}}\right) \\
& =2 e^{\beta y_{2}+i \alpha y_{1}} \cos ^{2}\left(\arctan e^{\beta y_{2}+i \alpha y_{1}}\right) \\
& =\frac{2 e^{\beta y_{2}+i \alpha y_{1}}}{1+e^{2\left(\beta y_{2}+i \alpha y_{1}\right)}}=\frac{1}{\beta+i \alpha} \frac{Q}{\sqrt{2}} .
\end{aligned}
$$

Similarly, from this identity we have

$$
Q_{x}-(\beta+i \alpha) \cos \left(\frac{\widetilde{Q}}{\sqrt{2}}\right) Q=0,
$$

so that from (2.6) and (2.12),

$$
\begin{aligned}
\widetilde{Q}_{t}+ & (\beta+i \alpha)\left[Q_{x} \cos \left(\frac{\widetilde{Q}}{\sqrt{2}}\right)+\frac{Q^{2}}{\sqrt{2}} \sin \left(\frac{\widetilde{Q}}{\sqrt{2}}\right)\right] \\
& =-(\beta+i \alpha)^{2} Q+Q_{x}^{2} Q^{-1}+\frac{1}{2} Q^{3}=0 .
\end{aligned}
$$

So far, we have proved the following result.

Lemma 2.4. Let $Q$ be a complex-valued soliton profile with scaling parameters $\alpha, \beta>0$ and shifts $x_{1}, x_{2} \in \mathbb{R}$, such that (2.9) is not satisfied. Then we have

$$
\frac{Q}{\sqrt{2}}-(\beta+i \alpha) \sin \left(\frac{\widetilde{Q}}{\sqrt{2}}\right) \equiv 0,
$$

and

$$
\widetilde{Q}_{t}+(\beta+i \alpha)\left[Q_{x} \cos \left(\frac{\widetilde{Q}}{\sqrt{2}}\right)+\frac{Q^{2}}{\sqrt{2}} \sin \left(\frac{\widetilde{Q}}{\sqrt{2}}\right)\right] \equiv 0,
$$

where $\sin z$ and $\cos z$ are defined on the complex plane in the usual sense.

We finish with a simple computational lemma.

Lemma 2.5. Fix $x_{1}, x_{2}$ such that (2.9) is not satisfied. Then, for all $\alpha, \beta \neq 0$ we have

$$
\mathcal{N}:=\frac{1}{2} \int_{-\infty}^{x} Q^{2}=\frac{2(\beta+i \alpha) e^{2\left(\beta y_{2}+i \alpha y_{1}\right)}}{1+e^{2\left(\beta y_{2}+i \alpha y_{1}\right)}},
$$

and

$$
\frac{1}{2} \int_{\mathbb{R}} Q^{2}=2(\beta+i \alpha),
$$


no matter what are $x_{1}, x_{2}$. Finally, if $L_{1}:=\log \left(1+e^{2\left(\beta x_{2}+i \alpha x_{1}\right)}\right)$,

$$
\int_{0}^{x} \mathcal{N}=\log \left(1+e^{2\left(\beta y_{2}+i \alpha y_{1}\right)}\right)-L_{1} .
$$

Note that the previous formula is well-defined since $x_{1}$ and $x_{2}$ do not satisfy (2.9).

Proof. It is not difficult to check that (2.15) is satisfied. Note that

$$
\lim _{x \rightarrow-\infty}\left|\frac{2(\beta+i \alpha) e^{2\left(\beta y_{2}+i \alpha y_{1}\right)}}{1+e^{2\left(\beta y_{2}+i \alpha y_{1}\right)}}\right|=0 .
$$

Identity (2.16) is a consequence of the fact that

$$
\lim _{x \rightarrow+\infty} \frac{2(\beta+i \alpha) e^{2\left(\beta y_{2}+i \alpha y_{1}\right)}}{1+e^{2\left(\beta y_{2}+i \alpha y_{1}\right)}}=2(\beta+i \alpha) .
$$

Finally, (2.17) is easy to check.

\section{BÄCKLUND TRANSFORMATION FOR MKDV}

The previous properties (i.e., Lemma 2.4) are consequence of a deeper result. In what follows, we fix a primitive $\tilde{f}$ of $f$, i.e.,

$$
\tilde{f}_{x}:=f,
$$

where $f$ is assumed only in $L^{2}(\mathbb{R})$. Notice that even if $f=f(t, x)$ is a solution of $\mathrm{mKdV}$, then a corresponding term $\widetilde{f}(t, x)$ may be unbounded in space.

Definition 3.1 (See e.g. 25]). Let

$$
\left(u_{a}, u_{b}, v_{a}, v_{b}, m\right) \in H^{1}(\mathbb{R} ; \mathbb{C})^{2} \times H^{-1}(\mathbb{R} ; \mathbb{C})^{2} \times \mathbb{C} .
$$

We set

$$
G:=\left(G_{1}, G_{2}\right), \quad G=G\left(u_{a}, u_{b}, v_{a}, v_{b}, m\right),
$$

where

$$
G_{1}\left(u_{a}, u_{b}, v_{a}, v_{b}, m\right):=\frac{\left(u_{a}-u_{b}\right)}{\sqrt{2}}-m \sin \left(\frac{\widetilde{u}_{a}+\widetilde{u}_{b}}{\sqrt{2}}\right),
$$

and

$$
\begin{aligned}
& G_{2}\left(u_{a}, u_{b}, v_{a}, v_{b}, m\right):=v_{a}-v_{b} \\
& \quad+m\left[\left(\left(u_{a}\right)_{x}+\left(u_{b}\right)_{x}\right) \cos \left(\frac{\widetilde{u}_{a}+\widetilde{u}_{b}}{\sqrt{2}}\right)+\frac{\left(u_{a}^{2}+u_{b}^{2}\right)}{\sqrt{2}} \sin \left(\frac{\widetilde{u}_{a}+\widetilde{u}_{b}}{\sqrt{2}}\right)\right] .
\end{aligned}
$$

For the moment we do not specify the space where $G\left(u_{a}, u_{b}, v_{a}, v_{b}, m\right)$ takes place if $\left(u_{a}, u_{b}, v_{a}, v_{b}, m\right) \in H^{1}(\mathbb{R} ; \mathbb{C})^{2} \times H^{-1}(\mathbb{R} ; \mathbb{C})^{2} \times \mathbb{C}$. However, thanks to Lemma 2.4 we have the following result.

Lemma 3.2. Assume that $x_{1}$ and $x_{2}$ do not satisfy (2.9). We have

$$
G\left(Q, 0, \widetilde{Q}_{t}, 0, \beta+i \alpha\right) \equiv(0,0) .
$$


Note that the previous identity can be extended by zero to the case where $x_{1}$ and $x_{2}$ satisfy (2.9), in such a form that now $G\left(Q, 0, \widetilde{Q}_{t}, 0, \beta+i \alpha\right)$, as a function of $\left(x_{1}, x_{2}\right) \in \mathbb{R}^{2}$, is now well-defined and continuous everywhere.

In what follows we consider the invertibility of the Bäcklund transformation on complex-valued functions. See 21] for the statement involving the real-valued solitons in the sine-Gordon case and 28 for the case of nonlinear Schrödinger solitons.

Proposition 3.3. Let $X^{0}:=\left(u_{a}^{0}, u_{b}^{0}, v_{a}^{0}, v_{b}^{0}, m^{0}\right) \in H^{1}(\mathbb{R} ; \mathbb{C})^{2} \times H^{-1}(\mathbb{R} ; \mathbb{C})^{2} \times \mathbb{C}$ be such that

$$
\begin{gathered}
\operatorname{Re} m^{0}>0, \\
G\left(X^{0}\right)=(0,0), \\
\sin \left(\frac{\widetilde{u}_{a}^{0}+\widetilde{u}_{b}^{0}}{\sqrt{2}}\right) \in H^{1}(\mathbb{R} ; \mathbb{C}),
\end{gathered}
$$

and

$$
\lim _{-\infty}\left(\widetilde{u}_{a}^{0}+\widetilde{u}_{b}^{0}\right)=0, \quad \lim _{+\infty}\left(\widetilde{u}_{a}^{0}+\widetilde{u}_{b}^{0}\right)=\sqrt{2} \pi .
$$

Assume additionally that the $O D E$

$$
\mu_{x}^{0}-m^{0} \cos \left(\frac{\widetilde{u}_{a}^{0}+\widetilde{u}_{b}^{0}}{\sqrt{2}}\right) \mu^{0}=0
$$

has a smooth solution $\mu^{0}=\mu^{0}(x) \in \mathbb{C}$ satisfying

$$
\mu^{0} \in H^{1}(\mathbb{R} ; \mathbb{C}), \quad\left|\mu^{0}(x)\right|>0, \quad\left|\frac{\mu_{x}^{0}(x)}{\mu^{0}(x)}\right| \leq C,
$$

and

$$
\int_{\mathbb{R}} \sin \left(\frac{\widetilde{u}_{a}^{0}+\widetilde{u}_{b}^{0}}{\sqrt{2}}\right) \mu^{0} \neq 0
$$

Then there exist $\nu_{0}>0$ and $C>0$ such that the following is satisfied. For any $0<\nu<\nu_{0}$ and any $\left(u_{a}, v_{a}\right) \in H^{1}(\mathbb{R} ; \mathbb{C}) \times H^{-1}(\mathbb{R} ; \mathbb{C})$ satisfying

$$
\left\|u_{a}-u_{a}^{0}\right\|_{H^{1}(\mathbb{R} ; \mathbb{C})}<\nu
$$

$G$ is well-defined in a neighborhood of $X^{0}$ and there exists an unique $\left(u_{b}, v_{b}, m\right)$ defined in an open subset of $H^{1}(\mathbb{R}, \mathbb{C}) \times H^{-1}(\mathbb{R} ; \mathbb{C}) \times \mathbb{C}$ such that

$$
\begin{gathered}
G\left(u_{a}, u_{b}, v_{a}, v_{b}, m\right) \equiv(0,0), \\
\left\|\widetilde{u}_{a}+\widetilde{u}_{b}-\widetilde{u}_{a}^{0}-\widetilde{u}_{b}^{0}\right\|_{H^{2}(\mathbb{R} ; \mathbb{C})} \leq C \nu, \\
\left\|u_{b}-u_{b}^{0}\right\|_{H^{1}(\mathbb{R} ; \mathbb{C})}+\left|m-m^{0}\right|<C \nu, \\
\sin \left(\frac{\widetilde{u}_{a}+\widetilde{u}_{b}}{\sqrt{2}}\right) \in H^{1}(\mathbb{R} ; \mathbb{C}),
\end{gathered}
$$


and

$$
\lim _{-\infty}\left(\widetilde{u}_{a}+\widetilde{u}_{b}\right)=0, \quad \lim _{+\infty}\left(\widetilde{u}_{a}+\widetilde{u}_{b}\right)=\sqrt{2} \pi .
$$

Proof. Given $u_{a}, u_{b}, m$ and $v_{a}$ well-defined, $v_{b}$ is uniquely defined from (3.3). We solve for $u_{b}$ and $m$ now. We will use the Implicit Function Theorem.

We make a change of variables in order to specify a suitable range for $G$ and being able to prove (3.16). Define

$$
u_{c}:=u_{a}+u_{b}-u_{c}^{0}, \quad u_{c}^{0}:=u_{a}^{0}+u_{b}^{0} \in H^{1}(\mathbb{R} ; \mathbb{C}),
$$

and similar for $\widetilde{u}_{c}$ and $\widetilde{u}_{c}^{0}$ :

$$
\left(\widetilde{u}_{c}\right)_{x}=u_{c}, \quad\left(\widetilde{u}_{c}^{0}\right)_{x}=u_{c}^{0} .
$$

In what follows, we will look for a suitable $\widetilde{u}_{c}$ with decay, and then we find $u_{b}$. Indeed, note that given $u_{c}$ and $u_{a}, u_{b}$ can be easily obtained. Then, with a slight abuse of notation, we consider $G$ defined as follows:

$$
G=\left(G_{1}, G_{2}\right), \quad G=G\left(u_{a}, \widetilde{u}_{c}, v_{a}, v_{b}, m\right),
$$

and

$$
\begin{aligned}
G: H^{1}(\mathbb{R} ; \mathbb{C}) \times H^{2}(\mathbb{R} ; \mathbb{C}) \times H^{-1}(\mathbb{R} ; \mathbb{C})^{2} \times \mathbb{C} & \longrightarrow H^{1}(\mathbb{R} ; \mathbb{C}) \times H^{-1}(\mathbb{R} ; \mathbb{C}), \\
\left(u_{a}, \widetilde{u}_{c}, v_{a}, v_{b}, m\right) & \longmapsto G\left(u_{a}, \widetilde{u}_{c}, v_{a}, v_{b}, m\right)
\end{aligned}
$$

where, from (3.2),

$$
G_{1}\left(u_{a}, \widetilde{u}_{c}, v_{a}, v_{b}, m\right):=\frac{\left(2 u_{a}-u_{c}^{0}-u_{c}\right)}{\sqrt{2}}-m \sin \left(\frac{\widetilde{u}_{c}^{0}+\widetilde{u}_{c}}{\sqrt{2}}\right),
$$

and from 3.3 ,

$$
\begin{aligned}
& G_{2}\left(u_{a}, \widetilde{u}_{c}, v_{a}, v_{b}, m\right):=v_{a}-v_{b} \\
& \quad+m\left[\left(u_{c}^{0}+u_{c}\right)_{x} \cos \left(\frac{\widetilde{u}_{c}^{0}+\widetilde{u}_{c}}{\sqrt{2}}\right)+\frac{\left(u_{a}^{2}+\left(u_{c}^{0}+u_{c}-u_{a}\right)^{2}\right)}{\sqrt{2}} \sin \left(\frac{\widetilde{u}_{c}^{0}+\widetilde{u}_{c}}{\sqrt{2}}\right)\right] .
\end{aligned}
$$

Clearly $G$ as in (3.18)-(3.19) defines a $C^{1}$ functional in a small neighborhood of $X^{1}$ given by

$$
X^{1}:=\left(u_{a}^{0}, 0, v_{a}^{0}, v_{b}^{0}, m^{0}\right) \in H^{1}(\mathbb{R} ; \mathbb{C}) \times H^{2}(\mathbb{R} ; \mathbb{C}) \times H^{-1}(\mathbb{R} ; \mathbb{C})^{2} \times \mathbb{C},
$$

where $G$ is well-defined according to (3.6). Let us apply the Implicit Function Theorem at this point. From (3.18) we have to show that

$$
u_{c}+m^{0} \cos \left(\frac{\widetilde{u}_{c}^{0}}{\sqrt{2}}\right) \widetilde{u}_{c}=f-m \sin \left(\frac{\widetilde{u}_{c}^{0}}{\sqrt{2}}\right)
$$

has a unique solution $\left(\widetilde{u}_{c}, m\right)$ such that $\widetilde{u}_{c} \in H^{2}(\mathbb{R} ; \mathbb{C})$, for any $f \in H^{1}(\mathbb{R} ; \mathbb{C})$ with linear bounds. From (3.7) we have

$$
\lim _{x \rightarrow \pm \infty} \cos \left(\frac{\widetilde{u}_{c}^{0}}{\sqrt{2}}\right)=\mp 1
$$

so that we can assume

$$
\mu^{0}(x)=\exp \left(m^{0} \int_{0}^{x} \cos \left(\frac{\widetilde{u}_{c}^{0}}{\sqrt{2}}\right)\right)
$$


Note that $\mu^{0}$ decays exponentially in space as $x \rightarrow \pm \infty$. We have

$$
\mu^{0} u_{c}+\left(\mu^{0}\right)_{x} \widetilde{u}_{c}=\mu^{0}\left[f-m \sin \left(\frac{\widetilde{u}_{c}^{0}}{\sqrt{2}}\right)\right] .
$$

Using (3.10), we choose $m \in \mathbb{C}$ such that

$$
\int_{\mathbb{R}} \mu^{0}\left[f-m \sin \left(\frac{\widetilde{u}_{c}^{0}}{\sqrt{2}}\right)\right]=0,
$$

so that

$$
|m| \leq C\|f\|_{L^{2}(\mathbb{R} ; \mathbb{C})},
$$

with $C>0$ depending on the quantity $\left|\int_{\mathbb{R}} \mu^{0} \sin \left(\frac{\widetilde{u}_{c}^{0}}{\sqrt{2}}\right)\right| \neq 0$ and $\left\|\mu^{0}\right\|_{L^{2}(\mathbb{R} ; \mathbb{C})}$. We get

$$
\widetilde{u}_{c}=\frac{1}{\mu^{0}} \int_{-\infty}^{x} \mu^{0}\left[f-m \sin \left(\frac{\widetilde{u}_{c}^{0}}{\sqrt{2}}\right)\right] .
$$

Finally, note that we have $u_{c} \in H^{1}(\mathbb{R} ; \mathbb{C})$. Indeed, first of all, thanks to (3.22), (3.8) and (3.21),

$$
\lim _{x \rightarrow \pm \infty} \widetilde{u}_{c}=\lim _{x \rightarrow \pm \infty} \frac{\mu^{0}}{\mu_{x}^{0}}\left[f-m \sin \left(\frac{\widetilde{u}_{c}^{0}}{\sqrt{2}}\right)\right]=0 .
$$

Note that if $s \leq x \ll-1$, from (3.21) we get

$$
\left|\frac{\mu^{0}(s)}{\mu^{0}(x)}\right|=\left|\exp \left(-m^{0} \int_{s}^{x} \cos \left(\frac{\widetilde{u}_{c}^{0}}{\sqrt{2}}\right)\right)\right| \leq C e^{-\operatorname{Re} m^{0}(x-s)},
$$

so that we have for $x<0$ and $\operatorname{larg} \notin$

$$
\begin{aligned}
\left|\widetilde{u}_{c}(x)\right| & \leq C \int_{-\infty}^{x} e^{-\left(\operatorname{Re} m^{0}\right)(x-s)}\left|f(s)-m \sin \left(\frac{\widetilde{u}_{c}^{0}(s)}{\sqrt{2}}\right)\right| d s \\
& \leq C \mathbf{1}_{(-\infty, x]} e^{-\left(\operatorname{Re} m^{0}\right)(\cdot)} \star\left|f-m \sin \left(\frac{\widetilde{u}_{c}^{0}}{\sqrt{2}}\right)\right|, \quad \operatorname{Re} m^{0}>0 .
\end{aligned}
$$

A similar result holds for $x>0$ large, after using (3.22). Therefore, from the Young's inequality,

$$
\left\|\widetilde{u}_{c}\right\|_{L^{2}(\mathbb{R} ; \mathbb{C})} \leq C\left\|f-m \sin \left(\frac{\widetilde{u}_{c}^{0}}{\sqrt{2}}\right)\right\|_{L^{2}(\mathbb{R} ; \mathbb{C})} \leq C\|f\|_{L^{2}(\mathbb{R} ; \mathbb{C})},
$$

as desired. On the other hand,

$$
\left(\widetilde{u}_{c}\right)_{x}=\left[f-m \sin \left(\frac{\widetilde{u}_{c}^{0}}{\sqrt{2}}\right)\right]-\frac{\mu_{x}^{0}}{\left(\mu^{0}\right)^{2}} \int_{-\infty}^{x} \mu^{0}\left[f-m \sin \left(\frac{\widetilde{u}_{c}^{0}}{\sqrt{2}}\right)\right] .
$$

Since $\mu_{x}^{0} / \mu^{0}$ is bounded (see (3.9)), we have $\widetilde{u}_{c} \in H^{1}(\mathbb{R} ; \mathbb{C}$ ). Finally, it is easy to see that $\widetilde{u}_{c} \in H^{2}(\mathbb{R} ; \mathbb{C})$. Note that the constant involving the boundedness of the linear operator $f \mapsto \widetilde{u}_{c}$ depends on the $H^{1}$-norm of $\mu^{0}$, which blows up if (2.9) is satisfied.

It turns out that we can apply the Implict Function Theorem to the operator $G$ described in (3.18)-(3.19), such that (3.12) is satisfied, provided (3.11) holds.

\footnotetext{
${ }^{7}$ Note that $\left\|\mu^{0}\right\|_{L^{2}(\mathbb{R} ; \mathbb{C})}$ blows up as (2.9) is attained.

${ }^{8}$ Here the symbol $\star$ denotes convolution.
} 
First of all, let us prove (3.15) and (3.16). Note that $\tilde{u}_{c} \in H^{2}(\mathbb{R} ; \mathbb{C})$, so that (3.15) and (3.16) are easily obtained.

On the other hand, estimate (3.13) is equivalent to the estimate

$$
\left\|\tilde{u}_{c}\right\|_{H^{2}(\mathbb{R} ; \mathbb{C})} \leq C \nu .
$$

We will obtain this estimate using the almost linear character of the operator $G$ around the point $X^{1}$. Since $\tilde{u}_{c}$ satisfies the equation (3.18), we have

or

$$
\frac{\left(2\left(u_{a}-u_{a}^{0}\right)-\left(\widetilde{u}_{c}\right)_{x}\right)}{\sqrt{2}}-m\left[\sin \left(\frac{\widetilde{u}_{c}^{0}+\widetilde{u}_{c}}{\sqrt{2}}\right)-\sin \left(\frac{\widetilde{u}_{c}^{0}}{\sqrt{2}}\right)\right]=0,
$$

$\frac{\left(2\left(u_{a}-u_{a}^{0}\right)-\left(\widetilde{u}_{c}\right)_{x}\right)}{\sqrt{2}}-m\left[\sin \left(\frac{\widetilde{u}_{c}^{0}}{\sqrt{2}}\right)\left\{\cos \left(\frac{\widetilde{u}_{c}}{\sqrt{2}}\right)-1\right\}+\cos \left(\frac{\widetilde{u}_{c}^{0}}{\sqrt{2}}\right) \sin \left(\frac{\widetilde{u}_{c}}{\sqrt{2}}\right)\right]=0$.

From (3.11) we know that $\left\|u_{a}-u_{a}^{0}\right\|_{H^{1}(\mathbb{R} ; \mathbb{C})}<\nu$. Since $\widetilde{u}_{c}$ is already small in $H^{2}(\mathbb{R} ; \mathbb{C})$, it is small in $L^{\infty}(\mathbb{R} ; \mathbb{C})$, therefore we have

$$
\left|\sin \left(\frac{\widetilde{u}_{c}}{\sqrt{2}}\right)-\frac{\widetilde{u}_{c}}{\sqrt{2}}\right| \leq C\left|\widetilde{u}_{c}\right|^{3},
$$

and

$$
\left|\cos \left(\frac{\widetilde{u}_{c}}{\sqrt{2}}\right)-1\right| \leq C\left|\widetilde{u}_{c}\right|^{2}
$$

In other words,

$$
\left(\widetilde{u}_{c}\right)_{x}+m \cos \left(\frac{\widetilde{u}_{c}^{0}}{\sqrt{2}}\right) \widetilde{u}_{c}=O_{H^{1}(\mathbb{R} ; \mathbb{C})}(\nu)+O\left(\left|\widetilde{u}_{c}\right|^{2}\right) .
$$

If we define

$$
\mu(x):=\exp \left(m \int_{0}^{x} \cos \left(\frac{\widetilde{u}_{c}^{0}}{\sqrt{2}}\right)\right),
$$

which is exponentially decreasing in space, the solution to system satisfies

$$
\left|\widetilde{u}_{c}\right| \leq \mu^{-1} \int_{0}^{x} \mu\left[O_{H^{1}(\mathbb{R} ; \mathbb{C})}(\nu)+O\left(\left|\widetilde{u}_{c}\right|^{2}\right)\right]
$$

The proof is complete.

Later we will need a second invertibility theorem. This time we assume that $m$ is fixed, $u_{b} \sim u_{b}^{0}$ is known and we look for $u_{a} \sim u_{a}^{0}$ this time. Note that the positive sign in front of (3.2) will be essential for the proof, otherwise we cannot take $m$ fixed.

Proposition 3.4. Let $X^{0}=\left(u_{a}^{0}, u_{b}^{0}, v_{a}^{0}, v_{b}^{0}, m^{0}\right) \in H^{1}(\mathbb{R} ; \mathbb{C})^{2} \times H^{-1}(\mathbb{R}, \mathbb{C}) \times \mathbb{C}$ be such that (3.4), (3.5), (3.6) and (3.7) are satisfied. Assume additionally that the $O D E$

$$
\left(\mu^{1}\right)_{x}+m \cos \left(\frac{\widetilde{u}_{a}^{0}+\widetilde{u}_{b}^{0}}{\sqrt{2}}\right) \mu^{1}=0,
$$

has a smooth solution $\mu^{1}=\mu^{1}(x) \in \mathbb{C}$ satisfying

$$
\left|\mu^{1}(x)\right|>0, \quad\left|\frac{\mu_{x}^{1}(x)}{\mu^{1}(x)}\right| \leq C, \quad \frac{1}{\mu^{1}} \in H^{1}(\mathbb{R} ; \mathbb{C}),
$$


and $G$ is smooth in a small neighborhood of $X^{0}$. Then there exists $\nu_{1}>0$ and a fixed constant $C>0$ such that for all $0<\nu<\nu_{1}$ the following is satisfied. For any $\left(u_{b}, v_{b}, m\right) \in H^{1}(\mathbb{R} ; \mathbb{C}) \times H^{-1}(\mathbb{R} ; \mathbb{C}) \times \mathbb{C}$ such that

$$
\left\|u_{b}-u_{b}^{0}\right\|_{H^{1}(\mathbb{R} ; \mathbb{C})}+\left|m-m^{0}\right|<\nu
$$

$G$ is well-defined and there exist unique $\left(u_{a}, v_{a}\right) \in H^{1}(\mathbb{R}, \mathbb{C}) \times H^{-1}(\mathbb{R} ; \mathbb{C})$ such that

$$
\begin{gathered}
G\left(u_{a}, u_{b}, v_{a}, v_{b}, m\right) \equiv(0,0), \\
\int_{\mathbb{R}}\left(u_{a}-u_{b}\right)\left(\frac{1}{\mu^{1}}\right)_{x}=0 . \\
\left\|\widetilde{u}_{a}+\widetilde{u}_{b}-\widetilde{u}_{a}^{0}-\widetilde{u}_{b}^{0}\right\|_{H^{2}(\mathbb{R} ; \mathbb{C})} \leq C \nu, \\
\lim _{-\infty}\left(\widetilde{u}_{a}+\widetilde{u}_{b}\right)=0, \quad \lim _{+\infty}\left(\widetilde{u}_{a}+\widetilde{u}_{b}\right)=\sqrt{2} \pi,
\end{gathered}
$$

and

$$
\left\|u_{a}-u_{a}^{0}\right\|_{H^{1}(\mathbb{R} ; \mathbb{C})}<C \nu .
$$

Proof. Given $u_{a}, u_{b}$ and $v_{b}$ well-defined, $v_{a}$ is uniquely defined from (3.3). We solve for $u_{a}$ now.

We follow the ideas of the proof of Proposition 3.3. However, this time we consider $G$ defined in the opposite sense: using (3.17),

$$
\begin{aligned}
& G=\left(G_{3}, G_{4}\right), \quad G=G\left(\widetilde{u}_{c}, u_{b}, v_{a}, v_{b}, m\right), \\
& G: H^{2}(\mathbb{R} ; \mathbb{C}) \times H^{1}(\mathbb{R} ; \mathbb{C}) \times H^{-1}(\mathbb{R} ; \mathbb{C})^{2} \times \mathbb{C} \longrightarrow H^{1}(\mathbb{R} ; \mathbb{C}) \times H^{-1}(\mathbb{R} ; \mathbb{C}), \\
&\left(\widetilde{u}_{c}, u_{b}, v_{a}, v_{b}, m\right) \longmapsto G\left(\widetilde{u}_{c}, u_{b}, v_{a}, v_{b}, m\right)
\end{aligned}
$$

with

$$
\int_{\mathbb{R}}\left(\widetilde{u}_{c}\right)_{x}\left(\frac{1}{\mu^{1}}\right)_{x}=0
$$

where, from (3.2),

$$
G_{3}\left(\widetilde{u}_{c}, u_{b}, v_{a}, v_{b}, m\right):=\frac{\left(u_{c}^{0}+u_{c}-2 u_{b}\right)}{\sqrt{2}}-m \sin \left(\frac{\widetilde{u}_{c}^{0}+\widetilde{u}_{c}}{\sqrt{2}}\right),
$$

and from (3.3),

$$
\begin{aligned}
& G_{4}\left(\widetilde{u}_{c}, u_{b}, v_{a}, v_{b}, m\right):=v_{a}-v_{b} \\
& \quad+m\left[\left(u_{c}^{0}+u_{c}\right)_{x} \cos \left(\frac{\widetilde{u}_{c}^{0}+\widetilde{u}_{c}}{\sqrt{2}}\right)+\frac{\left(\left(u_{c}^{0}+u_{c}-u_{b}\right)^{2}+u_{b}^{2}\right)}{\sqrt{2}} \sin \left(\frac{\widetilde{u}_{c}^{0}+\widetilde{u}_{c}}{\sqrt{2}}\right)\right] .
\end{aligned}
$$

Clearly $G$ as in (3.31)-(3.32) defines a $C^{1}$ functional in a small neighborhood of $X^{2}$ given by

$$
X^{2}:=\left(0, u_{b}^{0}, v_{a}^{0}, v_{b}^{0}, m^{0}\right) \in H^{2}(\mathbb{R} ; \mathbb{C}) \times H^{1}(\mathbb{R} ; \mathbb{C}) \times H^{-1}(\mathbb{R} ; \mathbb{C})^{2} \times \mathbb{C},
$$

where $G$ is well-defined according to (3.6) and $G\left(X^{2}\right)=(0,0)$.

Fix $m$ close enough to $m^{0}$. Now we have to show that

$$
u_{c}-m \cos \left(\frac{\widetilde{u}_{c}^{0}}{\sqrt{2}}\right) \widetilde{u}_{c}=f
$$


has a unique solution $\widetilde{u}_{c}$ such that $u_{c} \in H^{2}(\mathbb{R} ; \mathbb{C})$, for any $f \in H^{1}(\mathbb{R} ; \mathbb{C})$. Indeed, consider $\mu^{1}$ given by (3.23). It is not difficult to check that (see conditions (3.4), (3.25) and (3.7))

$$
\begin{gathered}
\operatorname{Re} m>0, \\
\lim _{ \pm \infty} \cos \left(\frac{\widetilde{u}_{c}^{0}}{\sqrt{2}}\right)=\mp 1,
\end{gathered}
$$

and

$$
\mu^{1}=\exp \left(-m \int_{0}^{x} \cos \left(\frac{\widetilde{u}_{c}^{0}}{\sqrt{2}}\right)\right) .
$$

Note that from (3.35) and (3.4) we have that $\left|\mu^{1}(x)\right|$ is exponentially growing in space as $x \rightarrow \pm \infty$. We have from (3.34),

$$
\left(\mu^{1} \widetilde{u}_{c}\right)_{x}=\mu^{1} f
$$

so that, thanks to (3.24),

$$
\widetilde{u}_{c}=\frac{1}{\mu^{1}} \mu^{1}(0) \widetilde{u}_{c}(0)+\frac{1}{\mu^{1}} \int_{0}^{x} \mu^{1} f .
$$

Clearly $\lim _{ \pm \infty} \widetilde{u}_{c}=0$ for $f \in H^{1}(\mathbb{R} ; \mathbb{C})$. In order to ensure uniqueness, we ask for $\widetilde{u}_{c}$ satisfying

$$
\int_{\mathbb{R}} u_{c}\left(\frac{1}{\mu^{1}}\right)_{x}=0
$$

which is nothing but (3.30) and (3.26), which is justified by (3.24). Let us show that $\widetilde{u}_{c} \in L^{2}(\mathbb{R} ; \mathbb{C})$. We have for $x>0$ large

$$
\left|\widetilde{u}_{c}(x)\right| \leq C \int_{0}^{x} e^{-(\operatorname{Re} m)(x-s)}|f(s)| d s=C e^{-(\operatorname{Re} m)(\cdot)} \star|f|, \quad \operatorname{Re} m>0 .
$$

A similar estimate can be established if $x<0$. Therefore, using Young's inequality,

$$
\left\|\widetilde{u}_{c}\right\|_{L^{2}(\mathbb{R} ; \mathbb{C})} \leq C\|f\|_{L^{2}(\mathbb{R} ; \mathbb{C})},
$$

as desired. Now we check that $u_{c} \in H^{1}(\mathbb{R} ; \mathbb{C})$. Indeed, we have

$$
u_{c}=f-\frac{\mu_{x}^{1}}{\left(\mu^{1}\right)^{2}} \int_{0}^{x} \mu^{1} f .
$$

Since $\mu_{x}^{1} / \mu^{1}$ is bounded, we have proven that $u_{c} \in L^{2}(\mathbb{R} ; \mathbb{C})$. A new iteration proves that $u_{c} \in H^{1}(\mathbb{R} ; \mathbb{C})$. Estimates (3.27)-(3.28)-(3.29) are consequence of the Implicit Function Theorem and can be proved as in the previous Proposition. The proof is complete.

We finish this Section by pointing out the role played by the Bäcklund transformation in the $\mathrm{mKdV}$ dynamics. We recall the following standard result.

Theorem 3.5. Let $m \in \mathbb{C}$ be a fixed parameter, and $I \subset \mathbb{R}$ an open time interval. Assume that $u_{b} \in C\left(I ; H^{1}(\mathbb{R} ; \mathbb{C})\right)$ and solves (1.1), i.e.,

$$
\left(u_{b}\right)_{t}+\left(\left(u_{b}\right)_{x x}+u_{b}^{3}\right)_{x}=0
$$

in the $H^{1}$-sense. Define $v_{b}:=\int_{0}^{x}\left(u_{b}\right)_{t}$ as a distribution in $H^{-1}(\mathbb{R} ; \mathbb{C})$. Then for each $t \in I$ the corresponding solution $\left(u_{a}(t), v_{a}(t)\right)$ of (3.2) -3.3) for $m$ fixed, obtained in the space $H^{1}(\mathbb{R} ; \mathbb{C}) \times H^{-1}(\mathbb{R} ; \mathbb{C})$, satisfies the following:

(1) $u_{a} \in C\left(I ; H^{1}(\mathbb{R} ; \mathbb{C})\right)$;

(2) $\left(u_{a}\right)_{t}=\left(v_{a}\right)_{x}$ is well-defined in $H^{-2}(\mathbb{R} ; \mathbb{C})$; and 
(3) $u_{a}$ solves (1.1) in the $H^{1}$-sense.

Proof. The first step is an easy consequence of the continuous character of the solution map given by the implicit function theorem. By density we can assume $u_{b}(t) \in H^{3}(\mathbb{R} ; \mathbb{C})$. From (3.2) we have

$$
\left(u_{a}\right)_{x}-\left(u_{b}\right)_{x}=m \cos \left(\frac{\widetilde{u}_{a}+\widetilde{u}_{b}}{\sqrt{2}}\right)\left(u_{a}+u_{b}\right),
$$

and

$$
\left(u_{a}\right)_{x x}-\left(u_{b}\right)_{x x}=m \cos \left(\frac{\widetilde{u}_{a}+\widetilde{u}_{b}}{\sqrt{2}}\right)\left(\left(u_{a}\right)_{x}+\left(u_{b}\right)_{x}\right)-\frac{m}{\sqrt{2}} \sin \left(\frac{\widetilde{u}_{a}+\widetilde{u}_{b}}{\sqrt{2}}\right)\left(u_{a}+u_{b}\right)^{2} .
$$

Therefore, from (3.3) and (3.2),

$$
\begin{aligned}
v_{a}-v_{b} & =-\left(\left(u_{a}\right)_{x x}-\left(u_{b}\right)_{x x}\right)-\frac{m}{\sqrt{2}} \sin \left(\frac{\widetilde{u}_{a}+\widetilde{u}_{b}}{\sqrt{2}}\right)\left[\left(u_{a}+u_{b}\right)^{2}+\left(u_{a}^{2}+u_{b}^{2}\right)\right] \\
& =-\left(\left(u_{a}\right)_{x x}-\left(u_{b}\right)_{x x}\right)-\left(u_{a}-u_{b}\right)\left(u_{a}^{2}+u_{a} u_{b}+u_{b}^{2}\right) \\
& =-\left(\left(u_{a}\right)_{x x}+u_{a}^{3}\right)-\left(\left(u_{b}\right)_{x x}+u_{b}^{3}\right) .
\end{aligned}
$$

Since $\left(v_{b}\right)_{x}=\left(u_{b}\right)_{t}$, we have from (3.36) that $\left(v_{b}\right)_{x}+\left(\left(u_{b}\right)_{x x}+u_{b}^{3}\right)_{x}=0$. Therefore

$$
\left(v_{a}\right)_{x}+\left(\left(u_{a}\right)_{x x}+u_{a}^{3}\right)_{x}=0 .
$$

Finally, if $\left(u_{a}\right)_{t}=\left(v_{a}\right)_{x}$, we have that $u_{a}$ solves (1.1). In order to prove this result, we compute the time derivative in (3.2): we get

$$
\left(u_{a}\right)_{t}-\left(u_{b}\right)_{t}=m \cos \left(\frac{\widetilde{u}_{a}+\widetilde{u}_{b}}{\sqrt{2}}\right)\left(\left(\widetilde{u}_{a}\right)_{t}+\left(\widetilde{u}_{b}\right)_{t}\right)
$$

Note that given $u_{b}$, the solution $u_{a}$ is uniquely defined, thanks to the Implicit Function Theorem. Additionally, from 3.3.

$$
\begin{aligned}
& \left(v_{a}\right)_{x}-\left(v_{b}\right)_{x}+ \\
& \quad+m\left[\left(\left(u_{a}\right)_{x x}+\left(u_{b}\right)_{x x}\right) \cos \left(\frac{\widetilde{u}_{a}+\widetilde{u}_{b}}{\sqrt{2}}\right)\right. \\
& -\frac{1}{\sqrt{2}}\left(\left(u_{a}\right)_{x}+\left(u_{b}\right)_{x}\right)\left(u_{a}+u_{b}\right) \sin \left(\frac{\widetilde{u}_{a}+\widetilde{u}_{b}}{\sqrt{2}}\right) \\
& \quad+\sqrt{2}\left(u_{a}\left(u_{a}\right)_{x}+u_{b}\left(u_{b}\right)_{x}\right) \sin \left(\frac{\widetilde{u}_{a}+\widetilde{u}_{b}}{\sqrt{2}}\right) \\
& \left.+\frac{\left(u_{a}^{2}+u_{b}^{2}\right)}{2}\left(u_{a}+u_{b}\right) \cos \left(\frac{\widetilde{u}_{a}+\widetilde{u}_{b}}{\sqrt{2}}\right)\right]=0 .
\end{aligned}
$$

We use (3.2) and (3.3) in the previous identity we get

$$
\begin{aligned}
& \left(v_{a}\right)_{x}-\left(v_{b}\right)_{x}+ \\
& \quad+\left[m\left(\left(u_{a}\right)_{x x}+\left(u_{b}\right)_{x x}\right) \cos \left(\frac{\widetilde{u}_{a}+\widetilde{u}_{b}}{\sqrt{2}}\right)+\left(u_{a}^{2}-u_{a} u_{b}+u_{b}^{2}\right)\left(\left(u_{a}\right)_{x}-\left(u_{b}\right)_{x}\right)\right]=0 .
\end{aligned}
$$

Finally, we use (3.37) to obtain

$$
\left(v_{a}\right)_{x}-\left(v_{b}\right)_{x}+m \cos \left(\frac{\widetilde{u}_{a}+\widetilde{u}_{b}}{\sqrt{2}}\right)\left(\left(u_{a}\right)_{x x}+u_{a}^{3}+\left(u_{b}\right)_{x x}+u_{b}^{3}\right)=0
$$

so (3.36) and (3.38) imply

$$
\left(v_{a}\right)_{x}-\left(v_{b}\right)_{x}=m \cos \left(\frac{\widetilde{u}_{a}+\widetilde{u}_{b}}{\sqrt{2}}\right)\left(v_{a}+v_{b}\right),
$$


so that from (3.39) and the uniqueness we conclude.

\section{Dynamics of complex-Valued mKdV Solitons}

In what follows we will apply the results from the previous section in a neighborhood of the complex soliton at time equals zero. Define (cf. (2.1)),

$$
\widetilde{Q}^{0}:=\widetilde{Q}(x ; \alpha, \beta, 0,0),
$$

and similarly for $Q^{0}$ and $\widetilde{Q}_{t}^{0}$. Recall that from Lemma 2.2 the complex soliton $Q^{0}$ is everywhere well-defined since (2.9) is not satisfied. Finally, given any

$$
\widetilde{z}_{b}^{0} \in \dot{H}^{1}(\mathbb{R} ; \mathbb{C}),
$$

we define $z_{b}^{0}$ by the identity (see (3.1) for instance)

$$
z_{b}^{0}:=\left(\widetilde{z}_{b}^{0}\right)_{x},
$$

and in term of distributions,

$$
w_{b}^{0}:=-\left(\left(z_{b}^{0}\right)_{x x}+\left(z_{b}^{0}\right)^{3}\right) \in H^{-1}(\mathbb{R} ; \mathbb{C}) .
$$

Lemma 4.1. There exists $\nu_{0}>0$ and $C>0$ such that, for all $0<\nu<\nu_{0}$, the following holds. For all $z_{b}^{0} \in H^{1}(\mathbb{R} ; \mathbb{C})$ satisfying

$$
\left\|z_{b}^{0}\right\|_{H^{1}(\mathbb{R} ; \mathbb{C})}<\nu
$$

there exist unique $y_{a}^{0} \in H^{1}(\mathbb{R}, \mathbb{C}), y_{a}^{1} \in H^{-1}(\mathbb{R}, \mathbb{C})$ and $m \in \mathbb{C}$, of the form

$$
y_{a}^{0}(x)=y_{a}^{0}\left[z_{b}^{0}\right](x), \quad y_{a}^{1}(x)=y_{a}^{1}\left[z_{b}^{0}, w_{b}^{0}\right](x), \quad m:=\beta+i \alpha+q^{0},
$$

such that

$$
\begin{gathered}
\left\|y_{a}^{0}\right\|_{H^{1}(\mathbb{R} ; \mathbb{C})}+\left|q^{0}\right| \leq C \nu \\
\widetilde{z}_{a}^{0}+\widetilde{y}_{a}^{0} \in H^{2}(\mathbb{R} ; \mathbb{C}), 9
\end{gathered}
$$

and

$$
G\left(Q^{0}+z_{a}^{0}, y_{a}^{0}, \widetilde{Q}_{t}^{0}+w_{a}^{0}, y_{a}^{1}, m\right) \equiv(0,0)
$$

Proof. Let $Q^{0}$ be the soliton profile with parameters $\beta, \alpha$ and $x_{1}=x_{2}=0$ (cf. (4.1)). We apply Proposition (3.3) with

$$
u_{a}^{0}:=Q^{0}, \quad u_{b}^{0}:=0, \quad v_{a}^{0}:=\widetilde{Q}_{t}^{0}, \quad v_{b}^{0}:=0,
$$

and

$$
m^{0}:=\beta+i \alpha .
$$

Clearly $\widetilde{u}_{a}^{0}+\widetilde{u}_{b}^{0}=\widetilde{Q}_{0}$ satisfies (3.6)-(3.7). From (2.13) we have

$$
\left(Q^{0}\right)_{x}-(\beta+i \alpha) \cos \left(\frac{\widetilde{Q}^{0}}{\sqrt{2}}\right) Q^{0}=0, \quad Q^{0}(-\infty)=0,
$$

so that we have (cf. (3.8)- (3.9) )

$$
\mu^{0}=Q^{0}
$$

Clearly $Q^{0}$ is never zero. Moreover, $\left|\left(Q^{0}\right)^{-1} Q_{x}^{0}\right|$ is bounded on $\mathbb{R}$. Now we prove that

$$
\int_{\mathbb{R}} \sin \left(\frac{\widetilde{Q}^{0}}{\sqrt{2}}\right) Q^{0} \neq 0
$$

\footnotetext{
${ }^{9}$ Note that both $\widetilde{z}_{a}^{0}$ and $\widetilde{y}_{a}^{0}$ may be unbounded functions, but the addition is bounded on $\mathbb{R}$.
} 
From (2.13) and (2.16),

$$
\int_{\mathbb{R}} \sin \left(\frac{\widetilde{Q}^{0}}{\sqrt{2}}\right) Q^{0}=\frac{1}{\sqrt{2}(\beta+i \alpha)} \int_{\mathbb{R}}\left(Q^{0}\right)^{2}=\frac{4(\beta+i \alpha)}{\sqrt{2}(\beta+i \alpha)}=2 \sqrt{2} .
$$

Before continuing, we need some definitions. We denote

$$
\alpha^{*}:=\alpha+\operatorname{Im} q^{0}, \quad \beta^{*}:=\beta+\operatorname{Re} q^{0},
$$

such that $m$ in (4.3) satisfies

$$
m=\beta+i \alpha+q^{0}=\beta^{*}+i \alpha^{*} .
$$

Since $q^{0}$ is small, we have that $\beta^{*}$ and $\alpha^{*}$ are positive quantities. Similarly, define

$$
\delta^{*}:=\left(\alpha^{*}\right)^{2}-3\left(\beta^{*}\right)^{2}, \quad \gamma^{*}:=3\left(\alpha^{*}\right)^{2}-\left(\beta^{*}\right)^{2},
$$

and compare with (1.4).

Consider the profile $\widetilde{Q}$ introduced in (2.1). We consider, for all $t \in \mathbb{R}$, the complex soliton profile

$$
\widetilde{Q}^{*}(t, x):=\widetilde{Q}\left(x ; \alpha^{*}, \beta^{*}, \delta^{*} t+x_{1}, \gamma^{*} t+x_{2}\right),
$$

with $\delta^{*}$ and $\gamma^{*}$ defined in (4.7), $x_{1}$ and $x_{2}$ possibly depending on time, and

$$
Q^{*}(t, x):=\partial_{x} \widetilde{Q}^{*}(t, x) .
$$

It is not difficult to see that (see e.g. (1.10) )

$$
Q^{*}(t, x)=Q_{c}(x-c t-\hat{x}), \quad \sqrt{c}=\beta^{*}+i \alpha^{*}, \quad \hat{x} \in \mathbb{C},
$$

which is a complex-valued solution of mKdV 1.1). Technically, the complex soliton $Q^{*}(t)$ has velocity $-\gamma^{*}=\left(\beta^{*}\right)^{2}-3\left(\alpha^{*}\right)^{2}$, a quantity that is always smaller than the corresponding speed $\left(=\left(\beta^{*}\right)^{2}\right)$ of the associated real-valued soliton $Q_{\left(\beta^{*}\right)^{2}}$ obtained by sending $\alpha^{*}$ to zero. Finally, as in (2.6) we define

$$
\widetilde{Q}_{t}^{*}(t, x):=-\left(\beta^{*}+i \alpha^{*}\right)^{2} Q^{*}(t, x) .
$$

Lemma 4.2. Fix $\alpha, \beta>0$. Assume that $x_{1}, x_{2}$ are time dependent functions such that

$$
\left|x_{1}^{\prime}(t)\right|+\left|x_{2}^{\prime}(t)\right| \ll\left|\delta^{*}-\gamma^{*}\right|=2\left(\left(\alpha^{*}\right)^{2}+\left(\beta^{*}\right)^{2}\right) .
$$

Then there exists only a sequence of times $t_{k} \in \mathbb{R}, k \in \mathbb{Z}$ such that (2.9) is satisfied. In particular, $\left(t_{k}\right)$ is a sequence with no limit points.

Proof. Note that (2.9) reads now

$$
\left(\delta^{*}-\gamma^{*}\right) t_{k}+\left(x_{1}-x_{2}\right)\left(t_{k}\right)=\frac{\pi}{\alpha^{*}}\left(k+\frac{1}{2}\right) .
$$

Since from (4.7) $\delta^{*}-\gamma^{*}=-2\left(\left(\alpha^{*}\right)^{2}+\left(\beta^{*}\right)^{2}\right) \neq 0$, and using (4.10) and the Inverse Function Theorem applied for each $k$, we have the desired conclusion.

We conclude that $\widetilde{Q}^{*}$ and $Q^{*}$ defined in (4.8) and (4.9) are well-defined except for an isolated sequence of times $t_{k}$. We impose now the following condition:

$$
t \in \mathbb{R} \text { satisfies } t \neq t_{k} \text { for all } k \in \mathbb{Z} \text {. }
$$


In what follows we will solve the Cauchy problem associated to $\mathrm{mKdV}$ with suitable initial data. Indeed, we will assume that

$$
y_{a}^{0} \text { is a real-valued function, and } y_{a}^{0} \in H^{1}(\mathbb{R}) \text {. }
$$

We will need the following 19

Theorem 4.3 (Kenig, Ponce and Vega $[23]$ ). For any $y_{a}^{0} \in H^{1}(\mathbb{R})$, there exists a uniqu@11 solution $y_{a} \in C\left(\mathbb{R}, H^{1}(\mathbb{R})\right)$ to $m K d V$, and

$$
\sup _{t \in \mathbb{R}}\left\|y_{a}(t)\right\|_{H^{1}(\mathbb{R})} \leq C\left\|y_{a}^{0}\right\|_{H^{1}(\mathbb{R})},
$$

with $C>0$ independent of time. Moreover, the mass

$$
M\left[y_{a}\right](t):=\frac{1}{2} \int_{\mathbb{R}} y_{a}^{2}(t, x) d x=M\left[y_{a}^{0}\right],
$$

and energy

$$
E\left[y_{a}\right](t):=\frac{1}{2} \int_{\mathbb{R}}\left(y_{a}\right)_{x}^{2}(t, x) d x-\frac{1}{4} \int_{\mathbb{R}}\left(y_{a}\right)^{4}(t, x) d x=E\left[y_{a}^{0}\right]
$$

are conserved quantities.

Assume 4.12). Let $y_{a} \in C\left(\mathbb{R}, H^{1}(\mathbb{R})\right)$ denote the corresponding solution for $\mathrm{mKdV}$ with initial data $y_{a}^{0}$. Since $\left\|y_{a}^{0}\right\|_{H^{1}} \leq C \eta$, we have for a (maybe different) constant $C>0$,

$$
\sup _{t \in \mathbb{R}}\left\|y_{a}(t)\right\|_{H^{1}(\mathbb{R})} \leq C \eta .
$$

In particular, we can define, for all $t \in \mathbb{R}$,

$$
\widetilde{y}_{a}(t):=\int_{0}^{x} y_{a}(t, s) d s
$$

and

$$
\left(\widetilde{y}_{a}\right)_{t}(t):=-\left(\left(y_{a}\right)_{x x}(t)+y_{a}^{3}(t)\right) \in H^{-1}(\mathbb{R}),
$$

because $y_{a}(t) \in L^{p}(\mathbb{R})$ for all $p \geq 2$.

Lemma 4.4. Assume that a time $t \in \mathbb{R}$ and $y_{a}^{0}$ are such that (4.11) and (4.12) hold. Then there are unique $z_{b}=z_{b}(t) \in H^{1}(\mathbb{R} ; \mathbb{C})$ and $w_{b}=w_{b}(t) \in H^{-1}(\mathbb{R} ; \mathbb{C})$ such that for all $t \neq t_{k}$,

$$
\begin{gathered}
\widetilde{z}_{b}+\widetilde{y}_{a} \in H^{2}(\mathbb{R} ; \mathbb{C}), \\
\frac{1}{\sqrt{2}}\left(Q^{*}+z_{b}-y_{a}\right)=\left(\beta+i \alpha+q^{0}\right) \sin \left(\frac{\widetilde{Q}^{*}+\widetilde{z}_{b}+\widetilde{y}_{a}}{\sqrt{2}}\right),
\end{gathered}
$$

where $\widetilde{Q}$ and $Q$ are defined in (4.8) and (4.9). Moreover, we have

$$
\begin{aligned}
0=\widetilde{Q}_{t}^{*}+w_{b}-\left(\widetilde{y}_{a}\right)_{t} & \\
+\left(\beta+i \alpha+q^{0}\right)[ & \left(Q_{x}^{*}+\left(z_{b}\right)_{x}+\left(y_{a}\right)_{x}\right) \cos \left(\frac{\widetilde{Q}^{*}+\widetilde{z}_{b}+\widetilde{y}_{a}}{\sqrt{2}}\right) \\
& \left.+\frac{\left(\left(Q^{*}+z_{b}\right)^{2}+y_{a}^{2}\right)}{\sqrt{2}} \sin \left(\frac{\widetilde{Q}^{*}+\widetilde{z}_{b}+\widetilde{y}_{a}}{\sqrt{2}}\right)\right],
\end{aligned}
$$

\footnotetext{
${ }^{10} \mathrm{We}$ recall that this result is consequence of the local Cauchy theory and the conservation of mass and energy 4.13)-4.14).

${ }^{11}$ In a certain sense, see 23 .
} 
and for all $t \neq t_{k}$,

$$
\left\|z_{b}(t)\right\|_{H^{1}(\mathbb{R} ; \mathbb{C})}<C \nu,
$$

with $C$ divergent as $t$ approaches a $t_{k}$.

Proof. We will use Proposition 3.4. For that it is enough to recall that from (2.13) and (2.14), and for all $t \neq t_{k} 12$

$$
\frac{1}{\sqrt{2}} Q^{*}=\left(\beta+i \alpha+q^{0}\right) \sin \left(\frac{\widetilde{Q}^{*}}{\sqrt{2}}\right)
$$

and

$$
\widetilde{Q}_{t}^{*}+\left(\beta+i \alpha+q^{0}\right)\left[Q_{x}^{*} \cos \left(\frac{\widetilde{Q}^{*}}{\sqrt{2}}\right)+\frac{\left(Q^{*}\right)^{2}}{\sqrt{2}} \sin \left(\frac{\widetilde{Q}^{*}}{\sqrt{2}}\right)\right]=0,
$$

so that we can apply Proposition 3.4 at $X^{0}=\left(Q^{*}, 0, \widetilde{Q}_{t}^{*}, 0, m\right)$, where from (4.21) we have $m=\left(\beta+i \alpha+q^{0}\right)$. It is not difficult to see that the function $\mu^{1}$ in (3.23) is given by

$$
\mu^{1}=\left(Q^{*}\right)^{-1}
$$

and (3.24) is satisfied. Note that we require the estimate (4.15) in order to obtain (4.18)-(4.19). Finally, (4.20) is a direct consequence of (3.29) and the fact that $\left\|\mu^{1}\right\|_{H^{1}(\mathbb{R} ; \mathbb{C})}$ blows up as $t$ approaches some $t_{k}$.

Remark 4.1. Since from (4.4) we get

$$
\frac{1}{\sqrt{2}}\left(Q^{0}+z_{b}^{0}-y_{a}^{0}\right)=\left(\beta+i \alpha+q^{0}\right) \sin \left(\frac{\widetilde{Q}^{0}+{\widetilde{z_{b}}}^{0}+{\widetilde{y_{a}}}^{0}}{\sqrt{2}}\right)
$$

we have that (4.18) implies by uniqueness

$$
\left(Q^{*}+z_{b}-y_{a}\right)(t=0)=Q^{0}+z_{b}^{0}-y_{a}^{0},
$$

i.e.,

$$
\left(Q^{*}+z_{b}\right)(t=0)=Q^{0}+z_{b}^{0} .
$$

We are ready to prove a detailed version of Theorem 1.3 , a result on complexvalued solitons.

Theorem 4.5. There exists $\nu_{0}>0$ such that for all $0<\nu<\nu_{0}$ the following holds. Consider the initial data $u_{b}^{0}:=Q^{0}+z_{b}^{0} \in H^{1}(\mathbb{R} ; \mathbb{C})$, where

$$
\left\|z_{b}^{0}\right\|_{H^{1}(\mathbb{R} ; \mathbb{C})}<\nu \text {. }
$$

Assume in addition that the corresponding function $y_{a}^{0}$ given by Lemma 4.1 is realvalued and belongs to $H^{1}(\mathbb{R})$. Fix $\varepsilon_{0}>0$. Then for all $t$ such that $\left|t-t_{k}\right| \geq \varepsilon_{0}$, with $t_{k}$ defined in Lemma 4.2, the function $u_{b}=Q^{*}+z_{b}$ introduced in Lemma 4.4 is an $H^{1}$ complex valued solution of $m K d V$, it satisfies $\left(u_{b}\right)_{t}=\left(Q^{*}+z_{b}\right)_{t}=\left(\widetilde{Q_{t}^{*}}+w_{b}\right)_{x}$ and the following estimate holds

$$
\sup _{\left|t-t_{k}\right| \geq \varepsilon_{0}}\left\|u_{b}(t)-Q^{*}(t)\right\|_{H^{1}(\mathbb{R} ; \mathbb{C})} \leq C_{\varepsilon_{0}} \nu
$$

Remark 4.2. The quantity $\varepsilon_{0}>0$ is just an auxiliary parameter and it can be made as small as required; however the constant $C_{\varepsilon_{0}}$ in (4.22) becomes singular as $\varepsilon_{0}$ approaches zero.

\footnotetext{
${ }^{12}$ It is interesting to note that the shifts $x_{1}, x_{2}$ on $Q^{*}(t, x)$ cannot be modified, otherwise there is no continuity at $t=0$.
} 
Remark 4.3. In Corollary 6.5 we will prove that there is an open set in $H^{1}(\mathbb{R} ; \mathbb{C})$ leading to $y_{a}^{0}$ real-valued. The openness of this set will be a consequence of the Implicit Function Theorem.

Proof. We apply Lemma 4.1. Assuming (4.12) we have $y_{a}^{0}$ real-valued so that there is an $\mathrm{mKdV}$ dynamics $y_{a}(t)$ constructed in Theorem 4.3. Finally we apply Lemma 4.4 to obtain the dynamical function $Q^{*}(t)+z_{b}(t)$. Finally we conclude using Theorem 3.5 .

Now we will prove that the mass and energy

$$
\frac{1}{2} \int_{\mathbb{R}} u_{b}^{2}(t), \quad \frac{1}{2} \int_{\mathbb{R}}\left(u_{b}\right)_{x}^{2}(t)-\frac{1}{4} \int_{\mathbb{R}} u_{b}^{4}(t),
$$

remain conserved for all time, without using the mKdV equation (1.1), but only the Bäcklund transformation (4.18). The fact that $\widetilde{z}_{b}+\widetilde{y}_{a} \in H^{1}(\mathbb{R} ; \mathbb{C})$ will be essential for the proof.

Corollary 4.6. Assume that $t \neq t_{k}$ for all $k \in \mathbb{Z}$. Then the quantity

$$
\frac{1}{2} \int_{\mathbb{R}}\left(Q^{*}+z_{b}\right)^{2}(t)
$$

is well-defined and independent of time, and

$$
\frac{1}{2} \int_{\mathbb{R}}\left(Q^{*}+z_{b}\right)^{2}(t)=\frac{1}{2} \int_{\mathbb{R}}\left(y_{a}^{0}\right)^{2}+2\left(\beta+i \alpha+q^{0}\right) .
$$

Moreover, (4.25) can be extended in a continuous form to every $t \in \mathbb{R}$.

Proof. Using (4.18) and multiplying each side by $\frac{1}{\sqrt{2}}\left(Q^{*}+z_{b}+y_{a}\right)$ we obtain

$$
\frac{1}{2}\left(Q^{*}+z_{b}-y_{a}\right)\left(Q^{*}+z_{b}-y_{a}\right)=-\left(\beta+i \alpha+q^{0}\right)\left[\cos \left(\frac{\widetilde{Q}^{*}+\widetilde{z}_{b}+\widetilde{y}_{a}}{\sqrt{2}}\right)\right]_{x} .
$$

We integrate on $\mathbb{R}$ to obtain

$$
\frac{1}{2} \int_{\mathbb{R}}\left(Q^{*}+z_{b}-y_{a}\right)\left(Q^{*}+z_{b}-y_{a}\right)=-\left.\left(\beta+i \alpha+q^{0}\right) \cos \left(\frac{\widetilde{Q}^{*}+\widetilde{z}_{b}+\widetilde{y}_{a}}{\sqrt{2}}\right)\right|_{-\infty} ^{\infty} .
$$

Since $\lim _{-\infty} \widetilde{Q}^{*}=0, \lim _{+\infty} \widetilde{Q}^{*}=\sqrt{2} \pi($ see (2.3) $)$ and $\lim _{ \pm \infty}\left(\widetilde{z}_{b}+\widetilde{y}_{a}\right)=0$, we get (4.24) - 4.25), because the mass of $y_{a}(t)$ is conserved.

Corollary 4.7. Assume that $t \neq t_{k}$ for all $k \in \mathbb{Z}$. Then the quantity

$$
E\left[Q^{*}+z_{b}\right](t):=\frac{1}{2} \int_{\mathbb{R}}\left(Q^{*}+z_{b}\right)_{x}^{2}(t)-\frac{1}{4} \int_{\mathbb{R}}\left(Q^{*}+z_{b}\right)^{4}(t)
$$

is well-defined and independent of time. Moreover, it satisfies

$$
\frac{1}{2} \int_{\mathbb{R}}\left(Q^{*}+z_{b}\right)_{x}^{2}(t)-\frac{1}{4} \int_{\mathbb{R}}\left(Q^{*}+z_{b}\right)^{4}(t)=E\left[y_{a}^{0}\right]-\frac{2}{3}\left(\beta^{*}+i \alpha^{*}\right)^{3} .
$$

Finally, this quantity can be extended in a continuous form to every $t \in \mathbb{R}$.

Proof. Denote $m=\left(\beta+i \alpha+q^{0}\right)$. From (4.18) we have

$$
\left(Q^{*}+z_{b}\right)_{x}-\left(y_{a}\right)_{x}=m \cos \left(\frac{\widetilde{Q}^{*}+\widetilde{z}_{b}+\widetilde{y}_{a}}{\sqrt{2}}\right)\left(Q^{*}+z_{b}+y_{a}\right)
$$


Multiplying by $\left(Q^{*}+z_{b}\right)_{x}+\left(y_{a}\right)_{x}$ we get

$$
\begin{aligned}
&\left(Q^{*}+z_{b}\right)_{x}^{2}-\left(y_{a}\right)_{x}^{2}=m \cos \left(\frac{\widetilde{Q}^{*}+\widetilde{z}_{b}+\widetilde{y}_{a}}{\sqrt{2}}\right)\left(Q^{*}+z_{b}+y_{a}\right)\left(Q^{*}+z_{b}+y_{a}\right)_{x} \\
&= m \cos \left(\frac{\widetilde{Q}^{*}+\widetilde{z}_{b}+\widetilde{y}_{a}}{\sqrt{2}}\right)\left[\left(Q^{*}+z_{b}\right)\left(Q^{*}+z_{b}\right)_{x}+y_{a}\left(y_{a}\right)_{x}\right. \\
&\left.y_{a}\left(Q^{*}+z_{b}\right)_{x}+\left(Q^{*}+z_{b}\right)\left(y_{a}\right)_{x}\right] .
\end{aligned}
$$

On the other hand, we multiply (4.27) by $y_{a}$ and $\left(Q^{*}+z_{b}\right)$ to obtain

$$
y_{a}\left(Q^{*}+z_{b}\right)_{x}-y_{a}\left(y_{a}\right)_{x}=m \cos \left(\frac{\widetilde{Q}^{*}+\widetilde{z}_{b}+\widetilde{y}_{a}}{\sqrt{2}}\right)\left(Q^{*}+z_{b}+y_{a}\right) y_{a}
$$

and

$$
\left(Q^{*}+z_{b}\right)\left(Q^{*}+z_{b}\right)_{x}-\left(Q^{*}+z_{b}\right)\left(y_{a}\right)_{x}=m \cos \left(\frac{\widetilde{Q}^{*}+\widetilde{z}_{b}+\widetilde{y}_{a}}{\sqrt{2}}\right)\left(Q^{*}+z_{b}+y_{a}\right)\left(Q^{*}+z_{b}\right) .
$$

If we subtract the latter from the former we get

$$
\begin{aligned}
y_{a}\left(Q^{*}+z_{b}\right)_{x}+\left(Q^{*}+z_{b}\right)\left(y_{a}\right)_{x}= & \left(Q^{*}+z_{b}\right)\left(Q^{*}+z_{b}\right)_{x}+y_{a}\left(y_{a}\right)_{x} \\
& +m \cos \left(\frac{\widetilde{Q}^{*}+\widetilde{z}_{b}+\widetilde{y}_{a}}{\sqrt{2}}\right)\left[y_{a}^{2}-\left(Q^{*}+z_{b}\right)^{2}\right] .
\end{aligned}
$$

Replacing (4.29) into (4.28),

$$
\begin{aligned}
\left(Q^{*}+z_{b}\right)_{x}^{2}-\left(y_{a}\right)_{x}^{2}= & m \cos \left(\frac{\widetilde{Q}^{*}+\widetilde{z}_{b}+\widetilde{y}_{a}}{\sqrt{2}}\right)\left[\left(Q^{*}+z_{b}\right)^{2}+y_{a}^{2}\right]_{x} \\
& +m^{2} \cos ^{2}\left(\frac{\widetilde{Q}^{*}+\widetilde{z}_{b}+\widetilde{y}_{a}}{\sqrt{2}}\right)\left[y_{a}^{2}-\left(Q^{*}+z_{b}\right)^{2}\right]
\end{aligned}
$$

Finally we use once again (4.18). We multiply by $\left(Q^{*}+z_{b}+y_{a}\right)$ :

$$
\frac{1}{\sqrt{2}}\left[\left(Q^{*}+z_{b}\right)^{2}-y_{a}^{2}\right]=m \sin \left(\frac{\widetilde{Q}^{*}+\widetilde{z}_{b}+\widetilde{y}_{a}}{\sqrt{2}}\right)\left(Q^{*}+z_{b}+y_{a}\right) .
$$

Replacing in (4.30) we finally arrive to the following identity:

$$
\begin{aligned}
\left(Q^{*}+z_{b}\right)_{x}^{2}-\left(y_{a}\right)_{x}^{2}= \\
=m \cos \left(\frac{\widetilde{Q}^{*}+\widetilde{z}_{b}+\widetilde{y}_{a}}{\sqrt{2}}\right)\left[\left(Q^{*}+z_{b}\right)^{2}+y_{a}^{2}\right]_{x} \\
\quad-m^{3} \sqrt{2} \cos ^{2}\left(\frac{\widetilde{Q}^{*}+\widetilde{z}_{b}+\widetilde{y}_{a}}{\sqrt{2}}\right) \sin \left(\frac{\widetilde{Q}^{*}+\widetilde{z}_{b}+\widetilde{y}_{a}}{\sqrt{2}}\right)\left(Q^{*}+z_{b}+y_{a}\right) .
\end{aligned}
$$


The last term on the right hand side above can be recognized as a total derivative. After integration, we obtain

$$
\begin{aligned}
\int_{\mathbb{R}}\left[\left(Q^{*}+z_{b}\right)_{x}^{2}-\left(y_{a}\right)_{x}^{2}\right]= & m \int_{\mathbb{R}} \cos \left(\frac{\widetilde{Q}^{*}+\widetilde{z}_{b}+\widetilde{y}_{a}}{\sqrt{2}}\right)\left[\left(Q^{*}+z_{b}\right)^{2}+y_{a}^{2}\right]_{x} \\
& +\left.\frac{2}{3} m^{3} \cos ^{3}\left(\frac{\widetilde{Q}^{*}+\widetilde{z}_{b}+\widetilde{y}_{a}}{\sqrt{2}}\right)\right|_{-\infty} ^{+\infty} \\
= & \frac{m}{\sqrt{2}} \int_{\mathbb{R}} \sin \left(\frac{\widetilde{Q}^{*}+\widetilde{z}_{b}+\widetilde{y}_{a}}{\sqrt{2}}\right)\left(Q^{*}+z_{b}+y_{a}\right)\left[\left(Q^{*}+z_{b}\right)^{2}+y_{a}^{2}\right] \\
& -\frac{4}{3} m^{3} \\
= & \frac{1}{2} \int_{\mathbb{R}}\left[\left(Q^{*}+z_{b}\right)^{2}-y_{a}^{2}\right]\left[\left(Q^{*}+z_{b}\right)^{2}+y_{a}^{2}\right]-\frac{4}{3} m^{3} \\
= & \frac{1}{2} \int_{\mathbb{R}}\left[\left(Q^{*}+z_{b}\right)^{4}-y_{a}^{4}\right]-\frac{4}{3} m^{3} .
\end{aligned}
$$

Finally

$$
\frac{1}{2} \int_{\mathbb{R}}\left(Q^{*}+z_{b}\right)_{x}^{2}-\frac{1}{4} \int_{\mathbb{R}}\left(Q^{*}+z_{b}\right)^{4}=\frac{1}{2} \int_{\mathbb{R}}\left(y_{a}\right)_{x}^{2}-\frac{1}{4} \int_{\mathbb{R}} y_{a}^{4}-\frac{2}{3}\left(\beta+i \alpha+q^{0}\right)^{3} .
$$

Since the right hand side above is conserved for all time, we have proved (4.26).

\section{COMplex SOlitons Versus BREATHERS}

We introduce now the notion of breather profile. Given parameters $x_{1}, x_{2} \in \mathbb{R}$ and $\alpha, \beta>0$, we consider $y_{1}$ and $y_{2}$ defined in (2.2). Let $\tilde{B}$ be the kink profile

$$
\widetilde{B}=\widetilde{B}\left(x ; \alpha, \beta, x_{1}, x_{2}\right):=2 \sqrt{2} \arctan \left(\frac{\beta}{\alpha} \frac{\sin \left(\alpha y_{1}\right)}{\cosh \left(\beta y_{2}\right)}\right),
$$

and with a slight abuse of notation, we redefine

$$
B:=\widetilde{B}_{x} .
$$

Note that

$$
\widetilde{B}(-\infty)=\widetilde{B}(+\infty)=0
$$

and for $k \in \mathbb{Z}$,

$$
\left\{\begin{array}{l}
\widetilde{B}\left(x ; \alpha, \beta, x_{1}+\frac{k \pi}{\alpha}, x_{2}\right)=(-1)^{k} \widetilde{B}\left(x ; \alpha, \beta, x_{1}, x_{2}\right), \\
B\left(x ; \alpha, \beta, x_{1}+\frac{k \pi}{\alpha}, x_{2}\right)=(-1)^{k} B\left(x ; \alpha, \beta, x_{1}, x_{2}\right) .
\end{array}\right.
$$

Now we introduce the directions associated to the shifts $x_{1}$ and $x_{2}$. Given a breather profile of parameters $\alpha, \beta, x_{1}$ and $x_{2}$, we define

$$
\begin{aligned}
& B_{1}=B_{1}\left(x ; \alpha, \beta, x_{1}, x_{2}\right):=\partial_{x_{1}} B, \\
& B_{2}=B_{2}\left(x ; \alpha, \beta, x_{1}, x_{2}\right):=\partial_{x_{2}} B .
\end{aligned}
$$

and for $\delta$ and $\gamma$ defined in (1.4),

$$
\widetilde{B}_{t}:=\delta B_{1}+\gamma B_{2} .
$$


We also have

$$
\widetilde{B}_{t}+B_{x x}+B^{3}=0
$$

see 4 for a proof of this identity.

If $x_{1}$ or $x_{2}$ are time-dependent variables, we assume that the associated $B_{j}$ corresponds to the partial derivative with respect to the time-independent variable $x_{j}$, evaluated at $x_{j}(t)$.

In this Section we will prove that there is a deep interplay between complex solitons and breather profiles. We start with the following identities.

Lemma 5.1. Let $(B, Q)$ be a pair breather-soliton profiles with scaling parameters $\alpha, \beta>0$ and shifts $x_{1}, x_{2} \in \mathbb{R}$. Assume that (2.9) is not satisfied. Then we have

$$
\frac{(B-Q)}{\sqrt{2}}-(\beta-i \alpha) \sin \left(\frac{\widetilde{B}+\widetilde{Q}}{\sqrt{2}}\right) \equiv 0,
$$

and

$$
\widetilde{B}_{t}-\widetilde{Q}_{t}+(\beta-i \alpha)\left[\left(B_{x}+Q_{x}\right) \cos \left(\frac{\widetilde{B}+\widetilde{Q}}{\sqrt{2}}\right)+\frac{\left(B^{2}+Q^{2}\right)}{\sqrt{2}} \sin \left(\frac{\widetilde{B}+\widetilde{Q}}{\sqrt{2}}\right)\right] \equiv 0 .
$$

Proof. Let us assume (5.9) and prove (5.10). We have from (2.6) and (2.11)

$$
\widetilde{Q}_{t}=-(\beta+i \alpha)^{2} Q=-\left(Q_{x x}+Q^{3}\right) .
$$

Using (5.9) we have

$$
B_{x}-Q_{x}-(\beta-i \alpha)(B+Q) \cos \left(\frac{\widetilde{B}+\widetilde{Q}}{\sqrt{2}}\right)=0,
$$

and

$$
\begin{gathered}
B_{x x}-Q_{x x}-(\beta-i \alpha)\left(B_{x}+Q_{x}\right) \cos \left(\frac{\widetilde{B}+\widetilde{Q}}{\sqrt{2}}\right) \\
+(\beta-i \alpha) \frac{(B+Q)^{2}}{\sqrt{2}} \sin \left(\frac{\widetilde{B}+\widetilde{Q}}{\sqrt{2}}\right)=0,
\end{gathered}
$$

so that using once again (5.9) and (5.8)

$$
\begin{aligned}
\widetilde{B}_{t}-\widetilde{Q}_{t}+(\beta-i \alpha)\left[\left(B_{x}+Q_{x}\right) \cos \left(\frac{\widetilde{B}+\widetilde{Q}}{\sqrt{2}}\right)+\frac{\left(B^{2}+Q^{2}\right)}{\sqrt{2}} \sin \left(\frac{\widetilde{B}+\widetilde{Q}}{\sqrt{2}}\right)\right] \\
=-\left(B_{x x}+B^{3}\right)+Q_{x x}+Q^{3}+\left[B_{x x}-Q_{x x}+(\beta-i \alpha) \frac{(B+Q)^{2}}{\sqrt{2}} \sin \left(\frac{\widetilde{B}+\widetilde{Q}}{\sqrt{2}}\right)\right] \\
\quad+(\beta-i \alpha) \frac{\left(B^{2}+Q^{2}\right)}{\sqrt{2}} \sin \left(\frac{\widetilde{B}+\widetilde{Q}}{\sqrt{2}}\right) \\
\quad Q^{3}-B^{3}+\sqrt{2}(\beta-i \alpha)\left(B^{2}+Q^{2}+B Q\right) \sin \left(\frac{\widetilde{B}+\widetilde{Q}}{\sqrt{2}}\right) \\
=Q^{3}-B^{3}+\left(B^{2}+Q^{2}+B Q\right)(B-Q)=0 .
\end{aligned}
$$

The proof of (5.9) is a tedious but straightforward computation which deeply requires the nature of the breather and soliton profiles. For the proof of this result, see Appendix $\mathrm{A}$ 
Corollary 5.2. Under the assumptions of Lemma 5.1, for any $x \in \mathbb{R}$ one has

$$
\frac{(B-\bar{Q})}{\sqrt{2}}-(\beta+i \alpha) \sin \left(\frac{\widetilde{B}+\widetilde{\bar{Q}}}{\sqrt{2}}\right) \equiv 0 \quad \text { in } \mathbb{R},
$$

where $\bar{Q}$ is the complex-valued soliton with parameters $\beta$ and $-\alpha$.

In order to prove some results in the next Section, we need several additional identities.

Corollary 5.3. Under the assumptions of Lemma 5.1, for any $x \in \mathbb{R}$ one has

$$
\cos \left(\frac{\widetilde{B}+\widetilde{Q}}{\sqrt{2}}\right)=1-\frac{1}{2(\beta-i \alpha)} \int_{-\infty}^{x}\left(B^{2}-Q^{2}\right),
$$

and

$$
\lim _{x \rightarrow \pm \infty} \cos \left(\frac{\widetilde{B}+\widetilde{Q}}{\sqrt{2}}\right)(x)=\mp 1 .
$$

Proof. We multiply by $\frac{1}{\sqrt{2}}(B+Q)$ in (5.9). We get

$$
\frac{1}{2}\left(B^{2}-Q^{2}\right)-(\beta-i \alpha) \sin \left(\frac{\widetilde{B}+\widetilde{Q}}{\sqrt{2}}\right) \times \frac{1}{\sqrt{2}}(B+Q)=0,
$$

i.e.,

$$
\frac{1}{2}\left(B^{2}-Q^{2}\right)+(\beta-i \alpha) \partial_{x} \cos \left(\frac{\widetilde{B}+\widetilde{Q}}{\sqrt{2}}\right)=0 .
$$

Since from (2.1) and (5.1) one has

$$
\lim _{x \rightarrow-\infty} \cos \left(\frac{\widetilde{B}+\widetilde{Q}}{\sqrt{2}}\right)=1
$$

Therefore, after integration,

$$
\frac{1}{2} \int_{-\infty}^{x}\left(B^{2}-Q^{2}\right)+(\beta-i \alpha)\left[\cos \left(\frac{\widetilde{B}+\widetilde{Q}}{\sqrt{2}}\right)-1\right]=0,
$$

as desired.

Lemma 5.4. We have

$$
\begin{aligned}
\mathcal{M} & :=\frac{1}{2} \int_{-\infty}^{x} B^{2} \\
& =2 \beta\left[1+\frac{2 \alpha\left(\beta \sin \left(2 \alpha y_{1}\right)+\alpha \sinh \left(2 \beta y_{2}\right)\right)}{\alpha^{2}+\beta^{2}-\beta^{2} \cos \left(2 \alpha y_{1}\right)+\alpha^{2} \cosh \left(2 \beta y_{2}\right)}\right] .
\end{aligned}
$$

Proof. See e.g. [4.

The following result is not difficult to prove.

Corollary 5.5. We have

$$
\int_{0}^{x} \mathcal{M}=2 \beta x+\log \left(\alpha^{2}+\beta^{2}-\beta^{2} \cos \left(2 \alpha y_{1}\right)+\alpha^{2} \cosh \left(2 \beta y_{2}\right)\right)-L_{0},
$$

where

$$
L_{0}:=\log \left(\alpha^{2}+\beta^{2}-\beta^{2} \cos \left(2 \alpha x_{1}\right)+\alpha^{2} \cosh \left(2 \beta x_{2}\right)\right) .
$$


Corollary 5.6. Under the assumptions of Lemma 5.1, we have

$$
\begin{aligned}
-(\beta-i \alpha) \int_{0}^{x} \cos \left(\frac{\widetilde{B}+\widetilde{Q}}{\sqrt{2}}\right)= & (\beta+i \alpha) x \\
& +\log \left(\alpha^{2}+\beta^{2}-\beta^{2} \cos \left(2 \alpha y_{1}\right)+\alpha^{2} \cosh \left(2 \beta y_{2}\right)\right) \\
& -\log \left(1+e^{2 \beta y_{2}+2 i \alpha y_{1}}\right)-L_{0}+L_{1}
\end{aligned}
$$

with $L_{0}$ and $L_{1}$ defined in (5.11) and (2.17).

Proof. We have from Corollaries 5.3 and 5.5, and (2.17),

$$
\begin{aligned}
\int_{0}^{x} \cos \left(\frac{\widetilde{B}+\widetilde{Q}}{\sqrt{2}}\right)=x-\frac{1}{(\beta-i \alpha)} \int_{0}^{x}(\mathcal{M}-\mathcal{N}) \\
=x-\frac{1}{\beta-i \alpha}\left[2 \beta x+\log \left(\alpha^{2}+\beta^{2}-\beta^{2} \cos \left(2 \alpha y_{1}\right)+\alpha^{2} \cosh \left(2 \beta y_{2}\right)\right)\right. \\
\left.\quad-\log \left(1+e^{2\left(\beta y_{2}+i \alpha y_{1}\right)}\right)-L_{0}+L_{1}\right] \\
=-\frac{1}{\beta-i \alpha}\left[(\beta+i \alpha) x+\log \left(\alpha^{2}+\beta^{2}-\beta^{2} \cos \left(2 \alpha y_{1}\right)+\alpha^{2} \cosh \left(2 \beta y_{2}\right)\right)\right. \\
\left.\quad-\log \left(1+e^{2\left(\beta y_{2}+i \alpha y_{1}\right)}\right)-L_{0}+L_{1}\right],
\end{aligned}
$$

as desired.

Corollary 5.7. Assume that $x_{1}, x_{2} \in \mathbb{R}$ do not satisfy (2.9). Consider the function

$$
\begin{aligned}
\mu\left(x ; \alpha, \beta, x_{1}, x_{2}\right) & :=2 \sqrt{2} \alpha^{2} \beta^{2} \frac{\cosh \left(\beta y_{2}\right) \cos \left(\alpha y_{1}\right)+i \sinh \left(\beta y_{2}\right) \sin \left(\alpha y_{1}\right)}{\alpha^{2} \cosh ^{2}\left(\beta y_{2}\right)+\beta^{2} \sin ^{2}\left(\alpha y_{1}\right)} \\
& =\beta \widetilde{B}_{1}-i \alpha \widetilde{B}_{2} .
\end{aligned}
$$

Then we have

$$
\lim _{x \rightarrow \pm \infty} \mu(x)=0
$$

and

$$
\mu_{x}=(\beta-i \alpha) \cos \left(\frac{\widetilde{B}+\widetilde{Q}}{\sqrt{2}}\right) \mu .
$$

Proof. Identity (5.13) is trivial. Let us prove (5.14). First of all, note that (cf. (2.7))

$$
\beta \widetilde{Q}_{1}-i \alpha \widetilde{Q}_{2} \equiv 0
$$

On the other hand, from (5.9) we have

$$
\left(\widetilde{B}_{1}-\widetilde{Q}_{1}\right)_{x}-(\beta-i \alpha) \cos \left(\frac{\widetilde{B}+\widetilde{Q}}{\sqrt{2}}\right)\left(\widetilde{B}_{1}+\widetilde{Q}_{1}\right)=0 .
$$

Similarly,

$$
\left(\widetilde{B}_{2}-\widetilde{Q}_{2}\right)_{x}-(\beta-i \alpha) \cos \left(\frac{\widetilde{B}+\widetilde{Q}}{\sqrt{2}}\right)\left(\widetilde{B}_{2}+\widetilde{Q}_{2}\right)=0
$$


We have then

$$
\begin{aligned}
\mu_{x}= & \left(\beta \widetilde{B}_{1}-i \alpha \widetilde{B}_{2}\right)_{x} \\
= & (\beta-i \alpha) \cos \left(\frac{\widetilde{B}+\widetilde{Q}}{\sqrt{2}}\right) \mu \\
& +\left(\beta \widetilde{Q}_{1}-i \alpha \widetilde{Q}_{2}\right)_{x}+(\beta-i \alpha) \cos \left(\frac{\widetilde{B}+\widetilde{Q}}{\sqrt{2}}\right)\left(\beta \widetilde{Q}_{1}-i \alpha \widetilde{Q}_{2}\right) \\
= & (\beta-i \alpha) \cos \left(\frac{\widetilde{B}+\widetilde{Q}}{\sqrt{2}}\right) \mu
\end{aligned}
$$

The proof is complete.

Lemma 5.8. Assume that (2.9) does not hold. Then $\mu$ defined in (5.12) has no zeroes, i.e. $|\mu(x)|>0$ for all $x \in \mathbb{R}$.

Proof. From (5.12) we have $\mu(x)=0$ if and only if $\cos \left(\beta y_{1}\right)=0$ and $\sinh \left(\alpha y_{2}\right)=0$, i.e. from (2.10) we have that (2.9) is satisfied.

Now we consider the opposite case, where the sign in front of (5.14) is negative. We finish this section with the following result.

Lemma 5.9. Assume that (2.9) does not hold. Then

$$
\mu^{1}\left(x ; \alpha, \beta, x_{1}, x_{2}\right):=\frac{1}{\mu}\left(x ; \alpha, \beta, x_{1}, x_{2}\right),
$$

with $\mu$ defined in (5.12), is well-defined, it has no zeroes and satisfies

$$
\lim _{x \rightarrow \pm \infty}\left|\mu^{1}(x)\right|=+\infty
$$

and

$$
\mu_{x}^{1}=-(\beta-i \alpha) \cos \left(\frac{\widetilde{B}+\widetilde{Q}}{\sqrt{2}}\right) \mu^{1} .
$$

Proof. A direct consequence of Corollary 5.7 and Lemma 5.8 .

\section{Double BäCKLund transformation FOR MKdV}

Assume that $x_{1}$ and $x_{2}$ do not satisfy (2.9). Consider the breather and soliton profiles $B$ and $Q$ defined in (5.2) and (2.5), well-defined according to Lemma 2.2. From Lemma 5.1 we have the following result.

Lemma 6.1. We have, for all $x \in \mathbb{R}$,

$$
G\left(B, Q, \widetilde{B}_{t}, \widetilde{Q}_{t}, \beta-i \alpha\right)=(0,0) .
$$

Note that the previous identity can be extended by zero to the case where $x_{1}$ and $x_{2}$ satisfy (2.9), in such a form that now $G\left(B, Q, \widetilde{B}_{t}, \widetilde{Q}_{t}, \beta-i \alpha\right)$ as a function of $x_{1}$ and $x_{2}$ is well-defined and continuous everywhere in $\mathbb{R}^{2}$ (and identically zero).

Define (cf. (5.1)-(5.7)),

$$
\left\{\begin{array}{l}
\widetilde{B}^{0}(x ; \alpha, \beta):=\widetilde{B}(x ; \alpha, \beta, 0,0), \\
\widetilde{B}_{t}^{0}(x ; \alpha, \beta):=\delta \widetilde{B}_{1}(x ; \alpha, \beta, 0,0)+\gamma \widetilde{B}_{2}(x ; \alpha, \beta, 0,0), \\
B^{0}(x ; \alpha, \beta):=\partial_{x} \widetilde{B}(x ; \alpha, \beta, 0,0) .
\end{array}\right.
$$


Finally, for $z_{a}^{0} \in H^{1}(\mathbb{R})$ we define

$$
\omega_{a}^{0}:=-\left(\left(z_{a}^{0}\right)_{x x}+\left(z_{a}^{0}\right)^{3}\right) \in H^{-1}(\mathbb{R}) .
$$

In what follows we will use Lemma 6.1 and apply Propositions 3.3 and 3.4 in a neighborhood of the complex soliton and the breather at time zero. Recall that from Lemma 2.2 the complex soliton $Q^{0}$ is everywhere well-defined since (2.9) is not satisfied.

Lemma 6.2. There exists $\eta_{0}>0$ and a constant $C>0$ such that, for all $0<\eta<$ $\eta_{0}$, the following is satisfied. Assume that $z_{a}^{0} \in H^{1}(\mathbb{R})$ satisfies

$$
\left\|z_{a}^{0}\right\|_{H^{1}(\mathbb{R})}<\eta, \quad \omega_{a}^{0} \text { defined by } \underline{6.2} \text {. }
$$

Then there exist unique $z_{b}^{0} \in H^{1}(\mathbb{R}, \mathbb{C}), \omega_{b}^{0} \in H^{-1}(\mathbb{R} ; \mathbb{C})$ and $m_{1} \in \mathbb{C}$, of the form

$$
z_{b}^{0}(x)=z_{b}^{0}\left[z_{a}^{0}\right](x), \quad \omega_{b}^{0}(x)=\omega_{b}^{0}\left[z_{a}^{0}, \omega_{a}^{0}\right](x), \quad m_{1}=m_{1}\left[z_{a}^{0}\right]:=\beta-i \alpha+p^{0},
$$

such that

$$
\begin{gathered}
\left\|z_{b}^{0}\right\|_{H^{1}(\mathbb{R} ; \mathbb{C})}+\left|p^{0}\right| \leq C \eta, \\
\widetilde{z}_{a}+\widetilde{z}_{b} \in H^{2}(\mathbb{R} ; \mathbb{C}),
\end{gathered}
$$

and

$$
G\left(B^{0}+z_{a}^{0}, Q^{0}+z_{b}^{0}, \widetilde{B}_{t}^{0}+\omega_{a}^{0}, \widetilde{Q}_{t}^{0}+\omega_{b}^{0}, m_{1}\right) \equiv(0,0) .
$$

Proof. Let $Q^{0}$ and $B^{0}$ be the soliton and breather profiles defined in (4.1) and (6.1). We will apply Proposition 3.3 with

$$
u_{a}^{0}:=B^{0}, \quad u_{b}^{0}:=Q^{0}, \quad v_{a}^{0}:=\widetilde{B}_{t}^{0}, \quad v_{b}^{0}:=\widetilde{Q}_{t}^{0}, \quad m^{0}:=\beta+i \alpha .
$$

Clearly $\operatorname{Re} m^{0}=\beta>0$, so that (3.4) is satisfied. On the other hand, (3.5) is a consequence of Lemma 6.1. From (5.9) condition (3.6) reads

$$
\sin \left(\frac{\widetilde{B}^{0}+\widetilde{Q}^{0}}{\sqrt{2}}\right)=\frac{\left(B^{0}-Q^{0}\right)}{\sqrt{2}(\beta-i \alpha)} \in H^{1}(\mathbb{R} ; \mathbb{C}) .
$$

Condition (3.7) is clearly satisfied (see (2.3) and (5.3)). From Corollary 5.7 we have

$$
\mu^{0}=\beta\left(\widetilde{B}_{1}\right)^{0}-i \alpha\left(\widetilde{B}_{2}\right)^{0} .
$$

Note that from Lemmas 2.2 and $5.8 \mu^{0}$ has no zeroes in the complex plane and it is exponentially decreasing in space. Finally, let us show that

$$
\int_{\mathbb{R}} \mu^{0} \sin \left(\frac{\widetilde{B}^{0}+\widetilde{Q}^{0}}{\sqrt{2}}\right)=\frac{4 i \alpha \beta}{\beta-i \alpha} .
$$

First of all, we have from (5.15)

$$
\begin{gathered}
{\left[\beta\left(\widetilde{B}_{1}\right)^{0}-i \alpha\left(\widetilde{B}_{2}\right)^{0}\right] \sin \left(\frac{\widetilde{B}^{0}+\widetilde{Q}^{0}}{\sqrt{2}}\right)} \\
=\left[\beta\left(\widetilde{B}_{1}+\widetilde{Q}_{1}\right)^{0}-i \alpha\left(\widetilde{B}_{2}+\widetilde{Q}_{2}\right)^{0}\right] \sin \left(\frac{\widetilde{B}^{0}+\widetilde{Q}^{0}}{\sqrt{2}}\right) \\
+\left[-\beta\left(\widetilde{Q}_{1}\right)^{0}+i \alpha\left(\widetilde{Q}_{2}\right)^{0}\right] \sin \left(\frac{\widetilde{B}^{0}+\widetilde{Q}^{0}}{\sqrt{2}}\right) .
\end{gathered}
$$


Consequently,

$$
\begin{aligned}
& {\left[\beta\left(\widetilde{B}_{1}\right)^{0}-i \alpha\left(\widetilde{B}_{2}\right)^{0}\right] \sin \left(\frac{\widetilde{B}^{0}+\widetilde{Q}^{0}}{\sqrt{2}}\right)} \\
& \quad=-\left.\sqrt{2} \beta \partial_{x_{1}}\left[\cos \left(\frac{\widetilde{B}+\widetilde{Q}}{\sqrt{2}}\right)\right]\right|^{0}+\left.i \alpha \sqrt{2} \partial_{x_{2}}\left[\cos \left(\frac{\widetilde{B}+\widetilde{Q}}{\sqrt{2}}\right)\right]\right|^{0} .
\end{aligned}
$$

Therefore, if $R_{1}, R_{2}>0$ are independent of $x_{1}$ and $x_{2}$,

$$
\begin{aligned}
\int_{-R_{2}}^{R_{1}} & \mu^{0} \sin \left(\frac{\widetilde{B}^{0}+\widetilde{Q}^{0}}{\sqrt{2}}\right) \\
& =\sqrt{2} \int_{-R_{2}}^{R_{1}}\left\{-\left.\beta \partial_{x_{1}}\left[\cos \left(\frac{\widetilde{B}+\widetilde{Q}}{\sqrt{2}}\right)\right]\right|^{0}+\left.i \alpha \partial_{x_{2}}\left[\cos \left(\frac{\widetilde{B}+\widetilde{Q}}{\sqrt{2}}\right)\right]\right|^{0}\right\} \\
& =\left.\sqrt{2}\left\{-\beta \partial_{x_{1}} \int_{-R_{2}}^{R_{1}} \cos \left(\frac{\widetilde{B}+\widetilde{Q}}{\sqrt{2}}\right)+i \alpha \partial_{x_{2}} \int_{-R_{2}}^{R_{1}} \cos \left(\frac{\widetilde{B}+\widetilde{Q}}{\sqrt{2}}\right)\right\}\right|^{0} .
\end{aligned}
$$

Now we use Corollary 5.6 we have

$$
\begin{aligned}
& \partial_{x_{1}} \int_{-R_{2}}^{R_{1}} \cos \left(\frac{\widetilde{B}+\widetilde{Q}}{\sqrt{2}}\right) \\
& \quad=-\left.\frac{1}{\beta-i \alpha}\left[\frac{2 i \alpha e^{2 \beta y_{2}+2 i \alpha y_{1}}}{1+e^{2 \beta y_{2}+2 i \alpha y_{1}}}-\frac{2 \alpha \beta^{2} \sin \left(2 \alpha y_{1}\right)}{\alpha^{2}+\beta^{2}-\beta^{2} \cos \left(2 \alpha y_{1}\right)+\alpha^{2} \cosh \left(2 \beta y_{2}\right)}\right]\right|_{-R_{1}} ^{R_{2}} .
\end{aligned}
$$

We have that

$$
\lim _{R_{1}, R_{2} \rightarrow \infty} \partial_{x_{1}} \int_{-R_{2}}^{R_{1}} \cos \left(\frac{\widetilde{B}+\widetilde{Q}}{\sqrt{2}}\right)=-\frac{2 i \alpha}{\beta-i \alpha} .
$$

Similarly,

$$
\begin{aligned}
& \partial_{x_{2}} \int_{-R_{2}}^{R_{1}} \cos \left(\frac{\widetilde{B}+\widetilde{Q}}{\sqrt{2}}\right)= \\
& \quad=-\left.\frac{1}{\beta-i \alpha}\left[\frac{2 \beta e^{2 \beta y_{2}+2 i \alpha y_{1}}}{1+e^{2 \beta y_{2}+2 i \alpha y_{1}}}-\frac{2 \alpha^{2} \beta \sinh \left(2 \beta y_{2}\right)}{\alpha^{2}+\beta^{2}-\beta^{2} \cos \left(2 \alpha y_{1}\right)+\alpha^{2} \cosh \left(2 \beta y_{2}\right)}\right]\right|_{-R_{1}} ^{R_{2}},
\end{aligned}
$$

and

$$
\lim _{R_{1}, R_{2} \rightarrow \infty} \partial_{x_{2}} \int_{-R_{2}}^{R_{1}} \cos \left(\frac{\widetilde{B}+\widetilde{Q}}{\sqrt{2}}\right)=-\frac{2 \beta-4 \beta}{\beta-i \alpha}=\frac{2 \beta}{\beta-i \alpha} .
$$

Adding the previous identities, we finally obtain

$$
\int_{\mathbb{R}} \mu^{0} \sin \left(\frac{\widetilde{B}^{0}+\widetilde{Q}^{0}}{\sqrt{2}}\right)=\sqrt{2}\left[\frac{2 i \alpha \beta}{\beta-i \alpha}+\frac{2 i \alpha \beta}{\beta-i \alpha}\right]=\frac{4 i \alpha \beta}{\beta-i \alpha} \neq 0 .
$$

After applying Proposition 3.3, we conclude.

Now we address the following very important question: is $y_{a}^{0}$ given in Lemma 4.1 real-valued for all $x \in \mathbb{R}$ ? In general, it seems that the answer is negative; however, if $z_{a}^{0}$ in Lemma 6.2 is real-valued, and $z_{b}^{0}$ from Lemma 6.2 satisfies (4.2), then the corresponding function $y_{a}^{0}$ given in Lemma 4.1 is also real-valued. This property is a consequence of a deep result called permutability theorem, that we explain below.

First of all, from Lemma 6.2 we have

$$
\frac{1}{\sqrt{2}}\left(B^{0}+z_{a}^{0}-Q^{0}-z_{b}^{0}\right)=\left(\beta-i \alpha+p^{0}\right) \sin \left(\frac{\widetilde{B}^{0}+\widetilde{z}_{a}^{0}+\widetilde{Q}^{0}+\widetilde{z}_{b}^{0}}{\sqrt{2}}\right),
$$


for some small $p^{0} \in \mathbb{C}$, and

$$
\sin \left(\frac{\widetilde{B}^{0}+\widetilde{z}_{a}^{0}+\widetilde{Q}^{0}+\widetilde{z}_{b}^{0}}{\sqrt{2}}\right) \in H^{1}(\mathbb{R} ; \mathbb{C}) .
$$

Now, by taking $\eta_{0}$ smaller if necessary, such that $C \eta<\nu_{0}$ for all $0<\eta<\eta_{0}$, Lemma 4.1 also applies. We get

$$
\frac{1}{\sqrt{2}}\left(Q^{0}+z_{b}^{0}-y_{a}^{0}\right)=\left(\beta+i \alpha+q^{0}\right) \sin \left(\frac{\widetilde{Q}^{0}+\widetilde{z}_{b}^{0}+\widetilde{y}_{a}^{0}}{\sqrt{2}}\right),
$$

for some small $q^{0}$.

We need some auxiliary notation. Define

$$
\beta_{*}:=\beta+\operatorname{Re} p^{0}, \quad \alpha_{*}:=\alpha-\operatorname{Im} p^{0},
$$

such that

$$
\left.\beta-i \alpha+p^{0}=\beta_{*}-i \alpha_{*} . \quad(\text { Compare with (4.6) }) .\right)
$$

We also consider

$$
\widetilde{Q}_{*}^{0}:=\widetilde{Q}\left(\cdot ;-\alpha_{*}, \beta_{*}, 0,0\right), \quad Q_{*}^{0}:=Q\left(\cdot ;-\alpha_{*}, \beta_{*}, 0,0\right) .
$$

Note that since $p^{0}$ is small, we have that $Q_{*}^{0}$ and $\bar{Q}^{0}$ share the same properties, since

$$
\left\|Q_{*}^{0}-\bar{Q}^{0}\right\|_{H^{1}(\mathbb{R} ; \mathbb{C})} \leq C \eta
$$

Moreover, thanks to Lemma 2.4 applied to $Q_{*}^{0}$,

$$
\frac{1}{\sqrt{2}} Q_{*}^{0}=\left(\beta-i \alpha+p^{0}\right) \sin \left(\frac{\widetilde{Q}_{*}^{0}}{\sqrt{2}}\right) \text {. }
$$

Consequently, applying Proposition 3.4 starting at $y_{a}^{0}$, and using (6.6) we can define $z_{d}^{0}$ via the identity

$$
\frac{1}{\sqrt{2}}\left(\bar{Q}^{0}+z_{d}^{0}-y_{a}^{0}\right)=\left(\beta-i \alpha+p^{0}\right) \sin \left(\frac{\widetilde{\bar{Q}}^{0}+\widetilde{z}_{d}^{0}+\widetilde{y}_{a}^{0}}{\sqrt{2}}\right) .
$$

Similarly, using (4.6) and (6.1) we define

$$
\left(\widetilde{B}^{0}\right)^{*}:=\widetilde{B}^{0}\left(\cdot ; \alpha^{*}, \beta^{*}\right), \quad\left(B^{0}\right)^{*}:=B\left(\cdot ; \alpha^{*}, \beta^{*}\right),
$$

so that from Lemma (5.1) we have

$$
\frac{1}{\sqrt{2}}\left(\left(B^{0}\right)^{*}-\left(Q^{0}\right)^{*}\right)=\left(\beta^{*}-i \alpha^{*}\right) \sin \left(\frac{\left(\widetilde{B^{0}}\right)^{*}+\left(\widetilde{Q^{0}}\right)^{*}}{\sqrt{2}}\right),
$$

and applying Corollary 5.2 we get

$$
\frac{1}{\sqrt{2}}\left(\left(B^{0}\right)^{*}-\overline{\left(Q^{0}\right)^{*}}\right)=\left(\beta+i \alpha+q^{0}\right) \sin \left(\frac{\left(\widetilde{B^{0}}\right)^{*}+\overline{\left(\widetilde{Q^{0}}\right)^{*}}}{\sqrt{2}}\right) .
$$

Using that

$$
\left\|\left(B^{0}\right)^{*}-B^{0}\right\|_{H^{1}(\mathbb{R})} \leq C \eta, \quad\left\|\overline{\left(Q^{0}\right)^{*}}-\overline{Q^{0}}\right\|_{H^{1}(\mathbb{R} ; \mathbb{C})} \leq C \eta,
$$

we can use Proposition 3.4 to obtain

$$
\frac{1}{\sqrt{2}}\left(B^{0}+z_{c}^{0}-\bar{Q}^{0}-z_{d}^{0}\right)=\left(\beta+i \alpha+q^{0}\right) \sin \left(\frac{\widetilde{B}^{0}+\widetilde{z}_{c}^{0}+\widetilde{\bar{Q}}^{0}+\widetilde{z}_{d}^{0}}{\sqrt{2}}\right),
$$


for some $z_{c}^{0}$ small. Note that the coefficients $\left(\beta-i \alpha+p^{0}\right)$ and $\left(\beta+i \alpha+q^{0}\right)$ were left fixed this time 13 Note additionally that $z_{d}^{0}$ and $z_{c}^{0}$ are bounded functions. Now we can announce a permutability theorem [25, p. 246]. This is part of a more general result, standard in the mathematical physics literature, see [39] for a formal proof in the Korteweg-de Vries (KdV) case.

Theorem 6.3 (Permutability theorem). We have

$$
\widetilde{z}_{c}^{0} \equiv{\widetilde{z_{a}}}^{0} \text {. }
$$

In particular, $z_{c}^{0}$ is an $H^{1}$ real-valued function.

Proof. Define

$$
\begin{gathered}
u_{0}:=y_{a}^{0} ; \quad u_{1}:=Q^{0}+z_{b}^{0} ; \quad u_{2}:=\bar{Q}^{0}+z_{d}^{0} \\
u_{12}:=B^{0}+z_{a}^{0} ; \quad u_{21}:=B^{0}+z_{c}^{0}
\end{gathered}
$$

and

$$
\kappa_{1}:=\beta+i \alpha+q^{0}, \quad \kappa_{2}:=\beta-i \alpha+p^{0} .
$$

Since $p^{0}$ and $q^{0}$ are small quantities, we have $\kappa_{1} \neq \kappa_{2}$, and both are nonzero complex numbers. Equations (6.3)-(6.9) read now

$$
\begin{gathered}
\frac{\left(u_{1}-u_{0}\right)}{\sqrt{2}}=\kappa_{1} \sin \left(\frac{\widetilde{u}_{1}+\widetilde{u}_{0}}{\sqrt{2}}\right), \\
\frac{\left(u_{12}-u_{1}\right)}{\sqrt{2}}=\kappa_{2} \sin \left(\frac{\widetilde{u}_{12}+\widetilde{u}_{1}}{\sqrt{2}}\right), \\
\frac{\left(u_{2}-u_{0}\right)}{\sqrt{2}}=\kappa_{2} \sin \left(\frac{\widetilde{u}_{2}+\widetilde{u}_{0}}{\sqrt{2}}\right),
\end{gathered}
$$

and

$$
\frac{\left(u_{21}-u_{2}\right)}{\sqrt{2}}=\kappa_{1} \sin \left(\frac{\widetilde{u}_{21}+\widetilde{u}_{2}}{\sqrt{2}}\right) .
$$

Note that $u_{1}$ and $u_{2}$ are obtained via the Implicit Function Theorem and therefore there is an associated uniqueness property for solutions obtained in a small neighborhood of the breather. The idea is to prove that $\widetilde{u}_{21} \equiv \widetilde{u}_{12}$. Define $\widetilde{u}_{3}$ via the identity

$$
\frac{\widetilde{u}_{3}-\widetilde{u}_{1}}{2 \sqrt{2}}=-\arctan \left[\left(\frac{\kappa_{1}-\kappa_{2}}{\kappa_{1}+\kappa_{2}}\right) \tan \left(\frac{\widetilde{u}_{12}-\widetilde{u}_{0}}{2 \sqrt{2}}\right)\right] .
$$

Whenever $u_{1}=Q^{0}, u_{12}=B^{0}, u_{0}=0, \kappa_{1}=\beta+i \alpha$ and $\kappa_{2}=\beta-i \alpha$, we get from (1.2),

$$
\begin{aligned}
\frac{\tilde{u}_{3}-\tilde{Q}^{0}}{2 \sqrt{2}} & =-\arctan \left[i \frac{\alpha}{\beta} \tan \left(\frac{\tilde{B}^{0}}{2 \sqrt{2}}\right)\right]=-\arctan \left(i \frac{\sin (\alpha x)}{\cosh (\beta x)}\right) \\
& =-\arctan \left(\frac{e^{i \alpha x}-e^{-i \alpha x}}{e^{\beta x}+e^{-\beta x}}\right) .
\end{aligned}
$$

\footnotetext{
${ }^{13}$ Note that the order of the coefficients will be important.
} 
Therefore, using (2.1),

$$
\begin{aligned}
\tilde{u}_{3} & =2 \sqrt{2} \arctan \left(e^{(\beta+i \alpha) x}\right)-2 \sqrt{2} \arctan \left(\frac{e^{i \alpha x}-e^{-i \alpha x}}{e^{\beta x}+e^{-\beta x}}\right) \\
& =2 \sqrt{2} \arctan \left(\frac{e^{(\beta+i \alpha) x}-\frac{e^{i \alpha x}-e^{-i \alpha x}}{e^{\beta x}+e^{-\beta x}}}{1+e^{(\beta+i \alpha) x} \cdot \frac{\left(e^{i \alpha x}-e^{-i \alpha x}\right)}{e^{\beta x}+e^{-\beta x}}}\right) \\
& =2 \sqrt{2} \arctan \left(e^{(\beta-i \alpha) x}\right) \\
& =\widetilde{Q}^{0} .
\end{aligned}
$$

Consequently, under the smallness assumptions in (6.11)-6.13) (the open character of these sets is essential) we have that $\tilde{u}_{3}$ is still well-defined on the real line with values on the complex plane, and it is close to $\widetilde{\widetilde{Q}^{0}}$, as well as $\widetilde{u}_{2}$.

Let us find an equation for $\widetilde{u}_{3}$. As usual, define $u_{3}:=\left(\widetilde{u}_{3}\right)_{x}$. We claim that

$$
\frac{\left(u_{3}-u_{0}\right)}{\sqrt{2}}=\kappa_{2} \sin \left(\frac{\widetilde{u}_{3}+\widetilde{u}_{0}}{\sqrt{2}}\right)
$$

in other words, $\widetilde{u}_{3} \equiv \widetilde{u}_{2}$. Similarly, if $\widetilde{u}_{4}$ solves

$$
\frac{\widetilde{u}_{2}-\widetilde{u}_{4}}{2 \sqrt{2}}=-\arctan \left[\left(\frac{\kappa_{1}-\kappa_{2}}{\kappa_{1}+\kappa_{2}}\right) \tan \left(\frac{\widetilde{u}_{21}-\widetilde{u}_{0}}{2 \sqrt{2}}\right)\right],
$$

then

$$
\frac{\left(u_{4}-u_{0}\right)}{\sqrt{2}}=\kappa_{1} \sin \left(\frac{\widetilde{u}_{4}+\widetilde{u}_{0}}{\sqrt{2}}\right)
$$

which implies $\widetilde{u}_{4} \equiv \widetilde{u}_{1}$. Finally, from (6.16) and (6.18) we have $\widetilde{u}_{12} \equiv \widetilde{u}_{21}$, which proves (6.10). Even better, we have 14

$$
\tan \left(\frac{\widetilde{u}_{12}-\widetilde{u}_{0}}{2 \sqrt{2}}\right)=-\left(\frac{\kappa_{1}+\kappa_{2}}{\kappa_{1}-\kappa_{2}}\right) \tan \left(\frac{\widetilde{u}_{2}-\widetilde{u}_{1}}{2 \sqrt{2}}\right) .
$$

Now let us prove (6.17). First of all, denote

$$
\ell:=\frac{\kappa_{1}+\kappa_{2}}{\kappa_{1}-\kappa_{2}}
$$

We have from (6.16)

$$
\frac{\widetilde{u}_{12}-\widetilde{u}_{0}}{\sqrt{2}}=-2 \arctan \left[\ell \tan \left(\frac{\widetilde{u}_{3}-\widetilde{u}_{1}}{2 \sqrt{2}}\right)\right],
$$

so that

$$
u_{12}-u_{0}=\frac{-\ell\left(u_{3}-u_{1}\right) \sec ^{2}\left(\frac{\widetilde{u}_{3}-\widetilde{u}_{1}}{2 \sqrt{2}}\right)}{1+\ell^{2} \tan ^{2}\left(\frac{\widetilde{u}_{3}-\widetilde{u}_{1}}{2 \sqrt{2}}\right)} .
$$

We also check that

$$
\sin \left(\frac{\widetilde{u}_{12}-\widetilde{u}_{0}}{\sqrt{2}}\right)=\frac{-2 \ell \tan \left(\frac{\widetilde{u}_{3}-\widetilde{u}_{1}}{2 \sqrt{2}}\right)}{1+\ell^{2} \tan ^{2}\left(\frac{\widetilde{u}_{3}-\widetilde{u}_{1}}{2 \sqrt{2}}\right)},
$$

\footnotetext{
${ }^{14}$ Note that this identity is well-defined at one particular set of functions, then extended by continuity.
} 
and

$$
\cos \left(\frac{\widetilde{u}_{12}-\widetilde{u}_{0}}{\sqrt{2}}\right)=\frac{1-\ell^{2} \tan ^{2}\left(\frac{\widetilde{u}_{3}-\widetilde{u}_{1}}{2 \sqrt{2}}\right)}{1+\ell^{2} \tan ^{2}\left(\frac{\widetilde{u}_{3}-\widetilde{u}_{1}}{2 \sqrt{2}}\right)} .
$$

Replacing in (6.15) we obtain

$$
\begin{aligned}
-\ell \frac{\left(u_{3}-u_{1}\right)}{\sqrt{2}} \sec ^{2}\left(\frac{\widetilde{u}_{3}-\widetilde{u}_{1}}{2 \sqrt{2}}\right)= & \kappa_{1} \sin \left(\frac{\widetilde{u}_{1}+\widetilde{u}_{0}}{\sqrt{2}}\right)\left[1+\ell^{2} \tan ^{2}\left(\frac{\widetilde{u}_{3}-\widetilde{u}_{1}}{2 \sqrt{2}}\right)\right] \\
& +\kappa_{2} \sin \left(\frac{\widetilde{u}_{1}+\widetilde{u}_{0}}{\sqrt{2}}\right)\left[1-\ell^{2} \tan ^{2}\left(\frac{\widetilde{u}_{3}-\widetilde{u}_{1}}{2 \sqrt{2}}\right)\right] \\
& -2 \ell \kappa_{2} \cos \left(\frac{\widetilde{u}_{1}+\widetilde{u}_{0}}{\sqrt{2}}\right) \tan \left(\frac{\widetilde{u}_{3}-\widetilde{u}_{1}}{2 \sqrt{2}}\right) .
\end{aligned}
$$

Using (6.20) and (6.14) we have

$$
\begin{aligned}
u_{3}-u_{0}-\sqrt{2} \kappa_{1} \sin \left(\frac{\widetilde{u}_{1}+\widetilde{u}_{0}}{\sqrt{2}}\right)= & -\sqrt{2} \cos ^{2}\left(\frac{\widetilde{u}_{3}-\widetilde{u}_{1}}{2 \sqrt{2}}\right) \times \\
\times & {\left[\left(\kappa_{1}-\kappa_{2}\right) \sin \left(\frac{\widetilde{u}_{1}+\widetilde{u}_{0}}{\sqrt{2}}\right)\left(1+\ell \tan ^{2}\left(\frac{\widetilde{u}_{3}-\widetilde{u}_{1}}{2 \sqrt{2}}\right)\right)\right.} \\
& \left.\quad-2 \kappa_{2} \cos \left(\frac{\widetilde{u}_{1}+\widetilde{u}_{0}}{\sqrt{2}}\right) \tan \left(\frac{\widetilde{u}_{3}-\widetilde{u}_{1}}{2 \sqrt{2}}\right)\right],
\end{aligned}
$$

i.e., after some standard trigonometric simplifications,

$$
\begin{aligned}
u_{3}-u_{0} & =\sqrt{2} \kappa_{2} \sin \left(\frac{\widetilde{u}_{1}+\widetilde{u}_{0}}{\sqrt{2}}\right) \cos \left(\frac{\widetilde{u}_{3}-\widetilde{u}_{1}}{\sqrt{2}}\right)+\sqrt{2} \kappa_{2} \cos \left(\frac{\widetilde{u}_{1}+\widetilde{u}_{0}}{\sqrt{2}}\right) \sin \left(\frac{\widetilde{u}_{3}-\widetilde{u}_{1}}{2 \sqrt{2}}\right) \\
& =\sqrt{2} \kappa_{2} \sin \left(\frac{\widetilde{u}_{3}+\widetilde{u}_{0}}{\sqrt{2}}\right)
\end{aligned}
$$

as desired.

Another consequence of the previous result is the following equivalent result.

Corollary 6.4. We have

$$
z_{d}^{0} \equiv \overline{z_{b}^{0}} \quad \text { and } \quad p^{0}=\overline{q^{0}} .
$$

In other words, $\alpha^{*}=\alpha_{*}$ and $\beta^{*}=\beta_{*}$.

Proof. Note that $z_{a}^{0} \equiv z_{c}^{0}$. From (6.9) we have

$$
\frac{1}{\sqrt{2}}\left(B^{0}+z_{a}^{0}-Q^{0}-\overline{z_{d}^{0}}\right)=\left(\beta-i \alpha+\overline{q^{0}}\right) \sin \left(\frac{\widetilde{B}^{0}+\widetilde{z}_{a}^{0}+\widetilde{Q}^{0}+\overline{{\widetilde{z_{d}}}^{0}}}{\sqrt{2}}\right) .
$$

From (6.3) and the uniqueness of $z_{b}^{0}$ and $p^{0}$ as implicit functions of $z_{a}^{0}$, we conclude.

The key result of this paper is the following surprising property.

Corollary 6.5. The function $y_{a}^{0}$ is real-valued. Moreover, there is a small ball of data $z_{a}^{0}$ in $H^{1}(\mathbb{R})$ for which the corresponding data $z_{b}^{0}$ lies in an open set of $H^{1}(\mathbb{R} ; \mathbb{C})$. 
Proof. The second statement is a consequence of the Implicit Function Theorem. On the other hand, the first one is consequence of the permutability theorem. First of all, note that

$$
\overline{\left(\beta+i \alpha+q^{0}\right)}=\beta-i \alpha+p^{0}=\beta^{*}-i \alpha^{*} .
$$

Now from (6.19) we get

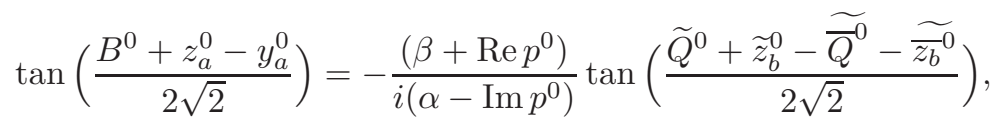

namely

$$
\tan \left(\frac{B^{0}+z_{a}^{0}-y_{a}^{0}}{2 \sqrt{2}}\right)=-\frac{\left(\beta+\operatorname{Re} p^{0}\right)}{\left(\alpha-\operatorname{Im} p^{0}\right)} \tanh \left(\frac{\operatorname{Im}\left(\widetilde{Q}^{0}+\widetilde{z}_{b}^{0}\right)}{\sqrt{2}}\right),
$$

from which we have $y_{a}^{0}(x)$ real-valued for all $x \in \mathbb{R}$.

The main advantage of the double Bäcklund transformation is that now the dynamics of $y_{a}^{0}$ is real-valued. We apply Theorem 4.5 with the initial data $z_{b}^{0}$ to get a complex solution of $\mathrm{mKdV} u_{b}(t)=Q^{*}(t)+z_{b}(t)$ defined for all $t \neq t_{k}$ and satisfying (4.22).

Now we reconstruct $z_{a}(t)$. As in (6.8), let us define, using (5.1), (4.6) and (4.7),

$$
\widetilde{B}^{*}(t, x):=\widetilde{B}\left(x ; \alpha^{*}, \beta^{*}, \delta^{*} t+x_{1}, \gamma^{*} t+x_{2}\right),
$$

and

$$
B^{*}(t, x)=\partial_{x} \widetilde{B}^{*}(t, x), \quad \widetilde{B}_{j}^{*}(t, x):=\left.\widetilde{B}_{j}\left(x ; \alpha^{*}, \beta^{*}, x_{1}, x_{2}\right)\right|_{x_{1}=\delta^{*} t+x_{1}, x_{2}=\gamma^{*} t+x_{2}} .
$$

In other words, we recover the original breather in (1.2) with scaling parameters $\alpha^{*}$ and $\beta^{*}$ and shifts $x_{1}, x_{2}$, provided they do not depend on time. Finally, as in (5.7) we define

$$
\widetilde{B}_{t}^{*}(t, x):=\delta \widetilde{B}_{1}^{*}(t, x)+\gamma \widetilde{B}_{2}^{*}(t, x) .
$$

Lemma 6.6. Assume that $t \in \mathbb{R}$ is such that (4.11) holds. Then there are unique $z_{a}=z_{a}(t) \in H^{1}(\mathbb{R} ; \mathbb{C})$ and $w_{a}=w_{a}(t) \in H^{-1}(\mathbb{R} ; \mathbb{C})$ such that

$$
\widetilde{z}_{a}+\widetilde{z}_{b} \in H^{2}(\mathbb{R} ; \mathbb{C}),
$$

$$
\frac{1}{\sqrt{2}}\left(B^{*}+z_{a}-Q^{*}-z_{b}\right)=\left(\beta-i \alpha+p^{0}\right) \sin \left(\frac{\widetilde{B}^{*}+\widetilde{z}_{a}+\widetilde{Q}^{*}+\widetilde{z}_{b}}{\sqrt{2}}\right),
$$

where $\widetilde{B}^{*}$ and $B^{*}$ are defined in (6.22) and (6.23). Moreover, we have

$$
\begin{aligned}
0=\widetilde{B}_{t}^{*}+w_{a}-\widetilde{Q}_{t}^{*}-w_{b} & \\
+\left(\beta-i \alpha+p^{0}\right) & {\left[\left(B_{x}^{*}+\left(z_{a}\right)_{x}+Q_{x}^{*}+\left(z_{b}\right)_{x}\right) \cos \left(\frac{\widetilde{B}^{*}+\widetilde{z}_{a}+\widetilde{Q}^{*}+\widetilde{z}_{b}}{\sqrt{2}}\right)\right.} \\
+ & \left.\frac{\left(\left(B^{*}+z_{a}\right)^{2}+\left(Q^{*}+z_{b}\right)^{2}\right)}{\sqrt{2}} \sin \left(\frac{\widetilde{B}^{*}+\widetilde{z}_{a}+\widetilde{Q}^{*}+\widetilde{z}_{b}}{\sqrt{2}}\right)\right],
\end{aligned}
$$

and for all $t \neq t_{k}$,

$$
\left\|z_{a}(t)\right\|_{H^{1}(\mathbb{R} ; \mathbb{C})} \leq C \eta
$$


Proof. We apply Proposition 3.4 at the point

$$
X^{0}:=\left(B^{*}, Q^{*}, \widetilde{B}_{t}^{*}, \widetilde{Q}_{t}^{*}, \beta-i \alpha+p^{0}\right),
$$

because a slight variation of Lemma 6.1 shows that (compare with (6.21)

$$
G\left(B^{*}, Q^{*}, \widetilde{B}_{t}^{*}, \widetilde{Q}_{t}^{*}, \beta-i \alpha+p^{0}\right)=(0,0) .
$$

Since $p^{0}$ is small,

$$
\operatorname{Re}\left(\beta-i \alpha+p^{0}\right)>0
$$

On the other hand, (3.6) is a consequence of (6.4). Similarly, from (2.3) we get (3.7) satisfied. Finally, in order to ensure that (3.24) is clearly satisfied, we apply Corollary 5.9 , we get

$$
\mu^{1}=\frac{1}{\mu^{*}}, \quad \text { where } \quad \mu^{*}:=\beta^{*} \widetilde{B}_{1}^{*}-i \alpha^{*} \widetilde{B}_{2}^{*} ;
$$

see Corollary 5.7 and (6.23). Then we conclude thanks to Proposition 3.4

Corollary 6.7. The function $z_{a}(t)$ as defined in (6.25) is real-valued.

Proof. The same proof as in Corollary 6.5 works mutatis mutandis, since now $y_{a}(t)$ is real-valued.

Proposition 6.8. For all $t \neq t_{k}, u_{a}=B^{*}+z_{a}$ is an $H^{1}$ real-valued solution to $m K d V$ with initial data $u_{0}$. Therefore, by uniqueness $15 B^{*}+z_{a} \equiv u$.

Proof. Since $u_{b}=Q^{*}+z_{b}$ solves mKdV, we use (6.25)-(6.26) and Theorem 3.5 to conclude.

\section{Stability of BREATHers}

In this final paragraph we prove Theorem 1.2, In what follows, we assume that $u_{0} \in H^{1}(\mathbb{R})$ satisfies $(1.5)$ for some $\eta$ small. Let $u \in C\left(\mathbb{R} ; H^{1}(\mathbb{R})\right)$ be the -unique in a certain sense- associated solution of the Cauchy problem (1.1), with initial data $u(0)=u_{0}$. Finally, we recall the conserved quantities mass (4.13) and energy (4.14).

Proof of Theorem 1.2, Consider $\varepsilon_{0}>0$ small but fixed, and $0<\eta<\eta_{0}$ small. From Lemmas 6.2 and 6.6 the proof is not difficult. Indeed, define the tubular neighborhood

$$
\mathcal{V}\left(A_{0}, \eta\right):=\left\{U \in H^{1}(\mathbb{R}) \mid \inf _{\tilde{x}_{1}, \tilde{x}_{2} \in \mathbb{R}}\left\|U-B\left(\cdot ; \alpha, \beta, \tilde{x}_{1}, \tilde{x}_{2}\right)\right\| \leq A_{0} \eta\right\} .
$$

Note that $B$ represents here the breather profile defined in (5.2). The original breather $B(t)$ from (1.2) can be recovered using (6.22) as follows (there is a slight abuse of notation here, but it is easily understood):

$$
B\left(t, x ; \alpha, \beta, x_{1}, x_{2}\right)=B\left(x ; \alpha, \beta, \delta t+x_{1}, \gamma t+x_{2}\right) .
$$

We will prove that if $u(t) \in \mathcal{V}\left(A_{0}, \eta\right)$ for $t \in\left[0, T_{0}\right]$, with $T_{0}>0$ and $\left|T_{0}-t_{k}\right|>\varepsilon_{0}$, for all $k \in \mathbb{Z}$, then

$$
u(t) \in \mathcal{V}\left(A_{0} / 2, \eta\right)
$$

\footnotetext{
${ }^{15}$ Technically, what we need is a result about unconditional uniqueness, however, from 22$]$ one can conclude that such a result is valid for $\mathrm{mKdV}$ on the line if we consider data with $H^{1}$ regularity.
} 
which proves the result for all positive times far from the points $t_{k}$. First of all, by taking $\eta_{0}>0$ smaller if necessary, and $\eta \in\left(0, \eta_{0}\right)$, we can ensure that there are unique $x_{1}(t), x_{2}(t) \in \mathbb{R}$, defined on $\left[0, T_{0}\right]$, and such that

$$
z(t, x):=u(t, x)-B\left(x ; \alpha, \beta, \delta t+x_{1}(t), \gamma t+x_{2}(t)\right)
$$

satisfies

$$
\int_{\mathbb{R}} z(t, x) B_{1}\left(x ; \alpha, \beta, \delta t+x_{1}(t), \gamma t+x_{2}(t)\right) d x=0,
$$

and

$$
\int_{\mathbb{R}} z(t, x) B_{2}\left(x ; \alpha, \beta, \delta t+x_{1}(t), \gamma t+x_{2}(t)\right) d x=0 .
$$

The directions $B_{1}$ and $B_{2}$ are defined in (5.5)-(5.6) (see 4 for a similar statement and its proof). Moreover, we have

$$
\|z(0)\|_{H^{1}(\mathbb{R})} \lesssim \eta
$$

and similar estimates for $x_{1}(0)$ and $x_{2}(0)$, with constants not depending on $A_{0}$ large. Therefore condition (2.9) is not satisfied. For the sake of simplicity, we can assume $x_{1}(0)=x_{2}(0)=0$, otherwise we perform a shift in space and time on the solution to set them equal zero.

Define $z_{a}^{0}:=z(0)$ and apply Lemma 6.2 and then Lemma 4.1 with the corresponding $z_{b}^{0}$ obtained from Lemma 6.2. We will obtain a real-valued seed $y_{a}^{0}$ small in $H^{1}(\mathbb{R})$. Note that the constants involved in each inversion do not depend on $A_{0}$. In particular, the differences between $\alpha$ and $\alpha^{*}$, and $\beta$ and $\beta^{*}$ are not depending on $A_{0}$ :

$$
\left|\alpha-\alpha^{*}\right|+\left|\beta-\beta^{*}\right| \lesssim \eta
$$

Next, we evolve the mKdV equation with initial data $y_{a}^{0}$. From Theorem 4.3 we have the bound (4.15) for the dynamics $y_{a}(t)$. On the other hand, decomposition (7.3)-(7.4) implies that

$$
\left|x_{1}^{\prime}(t)\right|+\left|x_{2}^{\prime}(t)\right| \lesssim A_{0} \eta,
$$

from which the set of points where condition (4.11) is not satisfied is still a countable set of isolated points (see Lemma 4.2).

Now we are ready to apply Lemmas 4.4 and 6.6 with parameters $\alpha^{*}, \beta^{*}$ and shifts $x_{1}(t)$ and $x_{2}(t)$ in (4.8), (4.9) and (6.22)-(6.23). In that sense, we have chosen a unique set of parameters for each fixed time $t$, and the $\mathrm{mKdV}$ solution that we choose is the same as the original $u(t)$. Indeed, just notice that at $t=0$, we have from (4.18) at $t=0$ and (6.5),

$$
\begin{aligned}
\frac{1}{\sqrt{2}}\left(Q^{*}(0)+z_{b}(0)-y_{a}^{0}\right) & =\left(\beta+i \alpha+q^{0}\right) \sin \left(\frac{\widetilde{Q}^{*}(0)+\widetilde{z}_{b}(0)+\widetilde{y}_{a}^{0}}{\sqrt{2}}\right), \\
\frac{1}{\sqrt{2}}\left(Q^{0}+z_{b}^{0}-y_{a}^{0}\right) & =\left(\beta+i \alpha+q^{0}\right) \sin \left(\frac{\widetilde{Q}^{0}+\widetilde{z}_{b}^{0}+\widetilde{y}_{a}^{0}}{\sqrt{2}}\right) .
\end{aligned}
$$

Using the uniqueness of the solution obtained by the Implicit function theorem in a neighborhood of the base point, we have

$$
z_{b}(0)=Q^{0}-Q^{*}(0)+z_{b}^{0} \sim z_{b}^{0} .
$$


Now we use (6.25) at $t=0$ and (6.3):

$\frac{1}{\sqrt{2}}\left(B^{*}(0)+z_{a}(0)-Q^{*}(0)-z_{b}(0)\right)=\left(\beta-i \alpha+p^{0}\right) \sin \left(\frac{\widetilde{B}^{*}(0)+\widetilde{z}_{a}(0)+\widetilde{Q}^{*}(0)+\widetilde{z}_{b}(0)}{\sqrt{2}}\right)$,

and

$$
\frac{1}{\sqrt{2}}\left(B^{0}+z_{a}^{0}-Q^{0}-z_{b}^{0}\right)=\left(\beta-i \alpha+p^{0}\right) \sin \left(\frac{\widetilde{B}^{0}+\widetilde{z}_{a}^{0}+\widetilde{Q}^{0}+\widetilde{z}_{b}^{0}}{\sqrt{2}}\right) .
$$

From (7.7), we have

$$
\frac{1}{\sqrt{2}}\left(B^{*}(0)+z_{a}(0)-Q^{0}-z_{b}^{0}\right)=\left(\beta-i \alpha+p^{0}\right) \sin \left(\frac{\widetilde{B}^{*}(0)+\widetilde{z}_{a}(0)+\widetilde{Q}^{0}+\widetilde{z}_{b}^{0}}{\sqrt{2}}\right) .
$$

Once again, since $B^{0}$ and $B^{*}(0)$ are close, using the uniqueness of the solution obtained via the Implicit function Theorem, we conclude that

$$
B^{*}(0)+z_{a}(0)=B^{0}+z_{a}^{0} .
$$

Since both initial data are the same, we conclude that the solution obtained via the Bäcklund transformation is $u(t)$.

Note that the constants involved in the inversions are not depending on $A_{0}$. We finally get

$$
\sup _{\left|t-t_{k}\right| \geq \varepsilon_{0}}\left\|u(t)-B^{*}(t)\right\|_{H^{1}(\mathbb{R})} \leq C_{0} \eta
$$

where

$$
B^{*}(t, x):=B\left(x ; \alpha^{*}, \beta^{*}, \delta^{*} t+x_{1}(t), \gamma^{*} t+x_{2}(t)\right) .
$$

Finally, from (7.5) and after redefining the shift parameters, we get the desired conclusion, since for $A_{0}$ large enough, we have $C_{0} \leq \frac{1}{2} A_{0}$.

Now we deal with the remaining case $t \sim t_{k}$. Fix $k \in \mathbb{Z}$. Note that $z_{a}=u-B^{*}$ satisfies the equation

$$
\left(z_{a}\right)_{t}+\left[\left(z_{a}\right)_{x x}+3\left(B^{*}\right)^{2} z_{a}+3 B^{*} z_{a}^{2}+z_{a}^{3}\right]_{x}+x_{1}^{\prime}(t) B_{1}^{*}+x_{2}^{\prime}(t) B_{2}^{*}=0,
$$

in the $H^{1}$-sense. In what follows, we will prove that, maybe taken $\varepsilon_{0}$ smaller but independent of $k$, we have

$$
\sup _{\left|t-t_{k}\right| \leq \varepsilon_{0}}\left\|u(t)-B^{*}(t)\right\|_{H^{1}(\mathbb{R})} \leq 4 A_{0} \eta .
$$

Since $A_{0}$ grows with $\varepsilon_{0}$ small, that implies that, after choosing $\eta_{0}$ smaller if necessary, such an operation can be performed without any risk.

In what follows, we assume that there is $T^{*} \in\left(t_{k}-\varepsilon_{0}, t_{k}+\varepsilon_{0}\right]$ such that, for all $t \in\left[t_{k}-\varepsilon_{0}, T^{*}\right]$

$$
\left\|z_{a}(t)\right\|_{H^{1}(\mathbb{R})} \leq 4 A_{0} \eta
$$

and $T^{*}$ is maximal in the sense of the above definition (i.e., there is no $T^{* *}>T^{*}$ satisfying the previous property). If $T^{*}=t_{k}+\varepsilon_{0}$, there is nothing to prove and (7.10) holds.

Assume $T^{*}<t_{k}+\varepsilon_{0}$. Now we consider the quantity

$$
\frac{1}{2} \int_{\mathbb{R}} z_{a}^{2}(t), \quad t \in\left[t_{0}-\varepsilon_{0}, T^{*}\right] .
$$


We have after (7.9),

$$
\begin{aligned}
\partial_{t} \frac{1}{2} \int_{\mathbb{R}} z_{a}^{2}(t)= & \int_{\mathbb{R}}\left(z_{a}\right)_{x}\left[3\left(B^{*}\right)^{2} z_{a}+3 B^{*} z_{a}^{2}+z_{a}^{3}\right](t) \\
& +x_{1}^{\prime}(t) \int_{\mathbb{R}} z_{a}(t) B_{1}^{*}+x_{2}^{\prime}(t) \int_{\mathbb{R}} z_{a}(t) B_{2}^{*} .
\end{aligned}
$$

Using (7.11) and (7.6), we have for some - explicit- fixed constant $C>0$ depending only on $\alpha, \beta$ and 4 , and $\eta_{0}$ even smaller if necessary,

$$
\left|\partial_{t} \frac{1}{2} \int_{\mathbb{R}} z_{a}^{2}(t)\right| \leq C A_{0}^{2} \eta^{2} .
$$

After integration in time and using (7.8), we have

$$
\int_{\mathbb{R}} z_{a}^{2}\left(T^{*}\right) \leq \int_{\mathbb{R}} z_{a}^{2}\left(t_{0}-\varepsilon_{0}\right)+C \varepsilon_{0} A_{0}^{2} \eta^{2} \leq 1.9 A_{0}^{2} \eta^{2},
$$

if $\varepsilon_{0}$ is small but fixed. A similar estimate can be obtained for $\left\|\left(z_{a}\right)_{x}(t)\right\|_{H^{1}(\mathbb{R})}$ by proving an estimate of the form

$$
\left|\partial_{t} \frac{1}{2} \int_{\mathbb{R}}\left(z_{a}\right)_{x}^{2}(t)\right| \leq C A_{0}^{2} \eta^{2}
$$

Therefore estimate (7.11) has been bootstrapped, which implies that $T^{*}=t_{0}+\varepsilon_{0}$. Note that the estimates do not depend on $k$, but only on the length of the intervals $\sim \varepsilon_{0} 16$

We conclude that there is $\tilde{A}_{0}>0$ fixed such that

$$
\sup _{t \in \mathbb{R}}\left\|u(t)-B^{*}(t)\right\|_{H^{1}(\mathbb{R})} \leq \tilde{A}_{0} \eta .
$$

Finally, estimates (1.6) and (1.7) are obtained from (7.6), and using the fact that $\alpha^{*}$ and $\beta^{*}$ are close to $\alpha$ and $\beta$ in terms of $C \eta$. The proof is complete.

Remark 7.1. From the proof and the results in 13 it is easy to realize that the evolution of breathers can be estimated in a polynomial form in time for any $s>\frac{1}{4}$, however, in order to make things simpler, we will not address this issue.

Corollary 7.1. We have for all $t \neq t_{k}$

$$
\begin{aligned}
\frac{1}{2} \int_{\mathbb{R}}\left(B^{*}+z_{a}\right)^{2}(t) & =\frac{1}{2} \int_{\mathbb{R}}\left(Q^{*}+z_{b}\right)^{2}(t)+2\left(\beta^{*}-i \alpha^{*}\right) \\
& =M\left[y_{a}^{0}\right]+4 \beta^{*} .
\end{aligned}
$$

Moreover, this identity can be extended to any $t \in \mathbb{R}$.

Proof. Same as Corollary 4.6 .

Finally, we recall that $\gamma^{*}=3\left(\alpha^{*}\right)^{2}-\left(\beta^{*}\right)^{2}$ and $E[u]=\frac{1}{2} \int_{\mathbb{R}} u_{x}^{2}-\frac{1}{4} \int_{\mathbb{R}} u^{4}$.

Corollary 7.2. Assume that $t \neq t_{k}$ for all $k \in \mathbb{Z}$. Then we have

$$
\begin{aligned}
E\left[B^{*}+z_{a}\right](t) & =E\left[Q^{*}+z_{b}\right](t)-\frac{4}{3}\left(\beta^{*}-i \alpha^{*}\right)^{3} \\
& =E\left[y_{a}^{0}\right]+\frac{4}{3} \beta^{*} \gamma^{*} .
\end{aligned}
$$

\footnotetext{
${ }^{16}$ Note that an argument involving the uniform continuity of the $\mathrm{mKdV}$ flow will not work in this particular case since the sequence of times $\left(t_{k}\right)$ is unbounded.
} 
Finally, this quantity can be extended in a continuous form to every $t \in \mathbb{R}$.

Proof. Same as Corollary 4.7 .

\section{Asymptotic Stability}

We finally prove Theorem 1.4 Note that for some $c_{0}>0$ depending on $\eta>0$,

$$
\lim _{t \rightarrow+\infty}\left\|y_{a}(t)\right\|_{H^{1}\left(x \geq c_{0} t\right)}=0 .
$$

This result can be obtained by adapting the proof for the soliton case in the Martel and Merle's paper [31]. Indeed, consider

$$
\phi(x):=\frac{K}{\pi} \arctan \left(e^{x / K}\right), \quad K>0,
$$

so that

$$
\lim _{-\infty} \phi=0, \quad \lim _{+\infty} \phi=1, \quad \phi^{\prime \prime \prime} \leq \frac{1}{K^{2}} \phi^{\prime}, \quad \phi^{\prime}>0 \text { on } \mathbb{R} .
$$

Fix $c_{0}, t_{0}>0$. Consider the quantities

$$
\begin{gathered}
I(t):=\frac{1}{2} \int_{\mathbb{R}} y_{a}^{2}(t) \phi\left(x-c_{0} t_{0}+\frac{1}{2} c_{0}\left(t_{0}-t\right)\right), \\
J(t):=\int_{\mathbb{R}}\left[\frac{1}{2}\left(y_{a}\right)_{x}^{2}(t)-\frac{1}{4} y_{a}^{4}(t)+\frac{1}{2} y_{a}^{2}(t)\right] \phi\left(x-c_{0} t_{0}+\frac{1}{2} c_{0}\left(t_{0}-t\right)\right) .
\end{gathered}
$$

It is not difficult to see that

$$
I^{\prime}(t)=-\frac{1}{4} c_{0} \int_{\mathbb{R}} y_{a}^{2} \phi^{\prime}(t)+\frac{1}{2} \int_{\mathbb{R}} y_{a}^{2} \phi^{\prime \prime \prime}(t)-\frac{3}{2} \int_{\mathbb{R}}\left(y_{a}\right)_{x}^{2} \phi^{\prime}(t)+\frac{3}{4} \int_{\mathbb{R}} y_{a}^{4} \phi^{\prime}(t),
$$

so that using (8.2), and if $c_{0}>0$ is small (but depending on $\eta$ smaller if necessary),

$$
I^{\prime}(t) \leq 0 .
$$

We have then

$$
I\left(t_{0}\right) \leq I(0)=\frac{1}{2} \int_{\mathbb{R}} y_{a}^{2}(0) \phi\left(x-c_{0} t_{0}\right),
$$

and

$$
\lim _{t \rightarrow+\infty} I(t)=0 .
$$

A similar result holds for $J(t)$, which proves 8.11.

Note that $\widetilde{z}_{b}+\widetilde{y}_{a} \in H^{2}(\mathbb{R} ; \mathbb{C})$ (see (4.17)). In what follows, we will prove that this function satisfies better estimates than $y_{a}$ and $z_{b}$ if $x$ is taken large.

Fix $t \neq t_{k}$ large, with $\left|t-t_{k}\right| \geq \varepsilon_{0}$. We use the notation

$$
\widetilde{z}_{c}:=\widetilde{y}_{a}+\widetilde{z}_{b} \text {. }
$$

Note that from (3.27) we have

$$
\left\|\widetilde{z}_{c}(t)\right\|_{H^{2}(\mathbb{R} ; \mathbb{C})} \leq C \nu
$$


with $C=C\left(\varepsilon_{0}\right)$ independent of time. From the Bäcklund transformation (4.18) we obtain

$$
\begin{aligned}
\left(\widetilde{z}_{c}\right)_{x}-2 y_{a} & =\sqrt{2}\left(\beta+i \alpha+q^{0}\right)\left[\sin \left(\frac{\widetilde{Q}^{*}+\widetilde{z}_{c}}{\sqrt{2}}\right)-\sin \left(\frac{\widetilde{Q}^{*}}{\sqrt{2}}\right)\right] \\
& =\sqrt{2}\left(\beta+i \alpha+q^{0}\right)\left[\sin \left(\frac{\widetilde{Q}^{*}}{\sqrt{2}}\right)\left\{\cos \left(\frac{\widetilde{z}_{c}}{\sqrt{2}}\right)-1\right\}+\sin \left(\frac{\widetilde{z}_{c}}{\sqrt{2}}\right) \cos \left(\frac{\widetilde{Q}^{*}}{\sqrt{2}}\right)\right] \\
& =Q^{*}\left\{\cos \left(\frac{\widetilde{z}_{c}}{\sqrt{2}}\right)-1\right\}+\sqrt{2} \sin \left(\frac{\widetilde{z}_{c}}{\sqrt{2}}\right) \frac{Q_{x}^{*}}{Q^{*}} .
\end{aligned}
$$

Assume now that $x>c_{0} t / 2$. Then we have for some fixed constant $c>0$,

$$
\left|\frac{Q_{x}^{*}}{Q^{*}}+m\right| \leq e^{-c x}, \quad m=\beta+i \alpha+q^{0}=\beta^{*}+i \alpha^{*},
$$

and

where

$$
\left(\widetilde{z}_{c}\right)_{x}+m \widetilde{z}_{c}=g
$$

$$
g:=Q^{*}\left\{\cos \left(\frac{\widetilde{z}_{c}}{\sqrt{2}}\right)-1\right\}+\sqrt{2}\left\{\sin \left(\frac{\widetilde{z}_{c}}{\sqrt{2}}\right)-\frac{\widetilde{z}_{c}}{\sqrt{2}}\right\} \frac{Q_{x}^{*}}{Q^{*}}+\widetilde{z}_{c}\left\{\frac{Q_{x}^{*}}{Q^{*}}+m\right\}+2 y_{a} .
$$

Solving the previous ODE we get

$$
\widetilde{z}_{c}(t, x)=\widetilde{z}_{c}\left(t, c_{0} t / 2\right) e^{-m\left(x-c_{0} t / 2\right)}+\int_{c_{0} t / 2}^{x} g(t, s) e^{-m(x-s)} d s,
$$

so that

$$
\left|\widetilde{z}_{c}(t, x)\right| \lesssim\left|\widetilde{z}_{c}\left(t, c_{0} t / 2\right)\right| e^{-\beta^{*}\left(x-c_{0} t / 2\right)}+\int_{c_{0} t / 2}^{x}|g(t, s)| e^{-\beta^{*}(x-s)} d s .
$$

From the Young's inequality we get

$$
\left\|\widetilde{z}_{c}(t)\right\|_{L^{2}\left(x \geq c_{0} t\right)} \lesssim\left|\widetilde{z}_{c}\left(t, c_{0} t / 2\right)\right| e^{-\beta^{*} c_{0} t / 2}+\|g(t)\|_{L^{2}\left(x \geq c_{0} t\right)} e^{-\beta^{*} c_{0} t}
$$

Clearly

$$
\left|\widetilde{z}_{c}\left(t, c_{0} t / 2\right)\right| \lesssim\left\|\widetilde{z}_{c}(t)\right\|_{H^{1}(\mathbb{R} ; \mathbb{C})} \leq C \nu, \quad\|g(t)\|_{L^{2}\left(x \geq c_{0} t\right)} \leq C \nu^{2}+C \nu e^{-c t}+o(1) .
$$

Passing to the limit, we obtain for all $T_{n} \rightarrow+\infty,\left|T_{n}-t_{k}\right| \geq \varepsilon_{0}$ for all $n$ and $k$,

$$
\lim _{n \rightarrow+\infty}\left\|\widetilde{z}_{c}\left(T_{n}\right)\right\|_{L^{2}\left(x \geq c_{0} T_{n}\right)}=0 .
$$

A similar result can be obtained in the case of $z_{c}$ and $\left(z_{c}\right)_{x}$. Finally, from (8.3) we get

$$
\lim _{n \rightarrow+\infty}\left\|z_{b}\left(T_{n}\right)\right\|_{H^{1}\left(x \geq c_{0} T_{n}\right)}=0 .
$$

Finally, we repeat the same strategy with (6.25) and (6.24) to obtain

$$
\lim _{t \rightarrow+\infty}\left\|z_{a}\left(T_{n}\right)\right\|_{H^{1}\left(x \geq c_{0} T_{n}\right)}=0 .
$$

Note that since the flow map is continuous in time with values in $H^{1}$, we can extend the result to any sequence $T_{n} \rightarrow+\infty$ by choosing an $\varepsilon_{0}>0$ smaller but still independent of $k$.

Proof of Corollary 1.5. Assume that for all $c_{0}>0$ small it is possible to find $\tilde{T}_{0}>0$ very large such that

$$
\left\|z_{a}\left(\tilde{T}_{0}\right)\right\|_{H^{1}(\mathbb{R})}<c_{0}\left\|z_{a}^{0}\right\|_{H^{1}(\mathbb{R})} .
$$


With no loss of generality we can assume $\tilde{T}_{0} \neq t_{k}$ for all $k$, otherwise we perturb $\tilde{T}_{0}$ and the previous inequality still holds true. We apply the Bäcklund transform twice to find

and therefore

$$
\left\|y_{a}\left(\tilde{T}_{0}\right)\right\|_{H^{1}(\mathbb{R})}<C c_{0}\left\|z_{a}^{0}\right\|_{H^{1}(\mathbb{R})},
$$

$$
\left\|y_{a}(0)\right\|_{H^{1}(\mathbb{R})}<C c_{0}\left\|z_{a}^{0}\right\|_{H^{1}(\mathbb{R})}
$$

for some constant $C>0$. Two consecutive inversions of the Bäcklund transform leads to

$$
0<\left\|z_{a}^{0}\right\|_{H^{1}(\mathbb{R})}<C c_{0}\left\|z_{a}^{0}\right\|_{H^{1}(\mathbb{R})},
$$

which is a contradiction if $c_{0}>0$ is chosen small enough.

\section{Appendix A. Proof of Lemma 5.1}

We will use the specific character of the breather and soliton profiles. Since (2.9) does hold, both $\widetilde{Q}$ and $Q$ are well-defined everywhere. We have

$$
\sin \left(\frac{\widetilde{B}+\widetilde{Q}}{\sqrt{2}}\right)=\sin \left(2\left(\arctan \Theta_{1}+\arctan \Theta_{2}\right)\right),
$$

where from (2.1) and (5.1),

$$
\Theta_{2}:=e^{\beta y_{2}+i \alpha y_{1}}, \quad \Theta_{1}:=\frac{\beta}{\alpha} \frac{\sin \left(\alpha y_{1}\right)}{\cosh \left(\beta y_{2}\right)} .
$$

We have

$$
\begin{aligned}
\sin \left(\frac{\widetilde{B}+\widetilde{Q}}{\sqrt{2}}\right)=2[ & \left.\sin \left(\arctan \Theta_{1}\right) \cos \left(\arctan \Theta_{2}\right)+\sin \left(\arctan \Theta_{2}\right) \cos \left(\arctan \Theta_{1}\right)\right] \\
\times & {\left[\cos \left(\arctan \Theta_{1}\right) \cos \left(\arctan \Theta_{2}\right)-\sin \left(\arctan \Theta_{1}\right) \sin \left(\arctan \Theta_{2}\right)\right] } \\
=2[ & \tan \left(\arctan \Theta_{1}\right) \cos ^{2}\left(\arctan \Theta_{1}\right) \cos ^{2}\left(\arctan \Theta_{2}\right) \\
& -\sin ^{2}\left(\arctan \Theta_{1}\right) \tan \left(\arctan \Theta_{2}\right) \cos ^{2}\left(\arctan \Theta_{2}\right) \\
& +\cos ^{2}\left(\arctan \Theta_{1}\right) \tan \left(\arctan \Theta_{2}\right) \cos ^{2}\left(\arctan \Theta_{2}\right) \\
& \left.-\sin ^{2}\left(\arctan \Theta_{2}\right) \tan \left(\arctan \Theta_{1}\right) \cos ^{2}\left(\arctan \Theta_{1}\right)\right] .
\end{aligned}
$$

Since

we have

$$
\sin ^{2}(\arctan (z))=\frac{z^{2}}{1+z^{2}}, \quad \cos ^{2}(\arctan (z))=\frac{1}{1+z^{2}},
$$

$$
\sin \left(\frac{\widetilde{B}+\widetilde{Q}}{\sqrt{2}}\right)=\frac{2\left(\Theta_{1}-\Theta_{1}^{2} \Theta_{2}+\Theta_{2}-\Theta_{2}^{2} \Theta_{1}\right)}{\left(1+\Theta_{1}^{2}\right)\left(1+\Theta_{2}^{2}\right)} .
$$

On the other hand

$$
\begin{aligned}
\frac{1}{\sqrt{2}}(B-Q) & =2 \partial_{x}\left(\arctan \Theta_{1}-\arctan \Theta_{2}\right) \\
& =2\left(\frac{\Theta_{1, x}}{1+\Theta_{1}^{2}}-\frac{\Theta_{2, x}}{1+\Theta_{2}^{2}}\right) \\
& =2 \frac{\left(1+\Theta_{2}^{2}\right) \Theta_{1, x}-\left(1+\Theta_{1}^{2}\right) \Theta_{2, x}}{\left(1+\Theta_{1}^{2}\right)\left(1+\Theta_{2}^{2}\right)} .
\end{aligned}
$$


Hence, collecting terms and factorizing, from (5.9) we are lead to prove that

$$
\left(1+\Theta_{2}^{2}\right) \Theta_{1, x}-\left(1+\Theta_{1}^{2}\right) \Theta_{2, x}-(\beta-i \alpha)\left(\Theta_{1}-\Theta_{1}^{2} \Theta_{2}+\Theta_{2}-\Theta_{2}^{2} \Theta_{1}\right)=0 .
$$

Now we perform some computations. We have from (2.1),

and

$$
\Theta_{2, x}=(\beta+i \alpha) \Theta_{2},
$$

$$
\alpha\left(\beta+i \alpha \Theta_{1}^{2}\right) \cosh ^{2}\left(\beta y_{2}\right)=\beta\left(\alpha \cosh ^{2}\left(\beta y_{2}\right)+i \beta \sin ^{2}\left(\alpha y_{1}\right)\right),
$$

$$
\Theta_{1, x}=\left(\frac{\beta}{\alpha} \frac{\sin \left(\alpha y_{1}\right)}{\cosh \left(\beta y_{2}\right)}\right)_{x}=\frac{\alpha \beta \cos \left(\alpha y_{1}\right) \cosh \left(\beta y_{2}\right)-\beta^{2} \sin \left(\alpha y_{1}\right) \sinh \left(\beta y_{2}\right)}{\alpha \cosh ^{2}\left(\beta y_{2}\right)},
$$

so that

$$
\Theta_{1, x}-(\beta-i \alpha) \Theta_{1}=\beta\left[\frac{\alpha e^{i \alpha y_{1}} \cosh \left(\beta y_{2}\right)-\beta e^{\beta y_{2}} \sin \left(\alpha y_{1}\right)}{\alpha \cosh ^{2}\left(\beta y_{2}\right)}\right]
$$

and

$$
\begin{aligned}
{\left[\Theta_{1, x}+(\beta-i \alpha) \Theta_{1}\right] \Theta_{2}^{2} } & =\beta\left[\frac{\alpha e^{-i \alpha y_{1}} \cosh \left(\beta y_{2}\right)+\beta e^{-\beta y_{2}} \sin \left(\alpha y_{1}\right)}{\alpha \cosh ^{2}\left(\beta y_{2}\right)}\right] e^{2\left(\beta y_{2}+i \alpha y_{1}\right)} \\
& =\beta \Theta_{2}\left[\frac{\alpha e^{\beta y_{2}} \cosh \left(\beta y_{2}\right)+\beta e^{i \alpha y_{1}} \sin \left(\alpha y_{1}\right)}{\alpha \cosh ^{2}\left(\beta y_{2}\right)}\right]
\end{aligned}
$$

Using (A.3), A.4 , A.5 and (A.6) we have

l.h.s. of $\mathrm{A} .2 \mathrm{C}=$

$$
\begin{aligned}
= & \left(1+\Theta_{2}^{2}\right) \Theta_{1, x}-2\left(\beta+i \alpha \Theta_{1}^{2}\right) \Theta_{2}-(\beta-i \alpha)\left(1-\Theta_{2}^{2}\right) \Theta_{1} \\
= & {\left[\Theta_{1, x}-(\beta-i \alpha) \Theta_{1}\right]+\left[\Theta_{1, x}+(\beta-i \alpha) \Theta_{1}\right] \Theta_{2}^{2}-2\left(\beta+i \alpha \Theta_{1}^{2}\right) \Theta_{2} } \\
= & \beta\left[\frac{\alpha e^{i \alpha y_{1}} \cosh \left(\beta y_{2}\right)-\beta e^{\beta y_{2}} \sin \left(\alpha y_{1}\right)}{\alpha \cosh ^{2}\left(\beta y_{2}\right)}\right]+ \\
& +\beta \Theta_{2}\left[\frac{\alpha e^{\beta y_{2}} \cosh \left(\beta y_{2}\right)+\beta e^{i \alpha y_{1}} \sin \left(\alpha y_{1}\right)-2 \alpha \cosh ^{2}\left(\beta y_{2}\right)-2 i \beta \sin ^{2}\left(\alpha y_{1}\right)}{\alpha \cosh ^{2}\left(\beta y_{2}\right)}\right] \\
= & \beta\left[\frac{\alpha e^{i \alpha y_{1}} \cosh \left(\beta y_{2}\right)-\beta e^{\beta y_{2}} \sin \left(\alpha y_{1}\right)}{\alpha \cosh ^{2}\left(\beta y_{2}\right)}\right]+ \\
& +\beta \Theta_{2}\left[\frac{-\alpha e^{-\beta y_{2}} \cosh \left(\beta y_{2}\right)+\beta e^{-i \alpha y_{1}} \sin \left(\alpha y_{1}\right)}{\alpha \cosh ^{2}\left(\beta y_{2}\right)}\right] \\
= & 0,
\end{aligned}
$$

which proves A.2.

\section{REFERENCES}

[1] M. Ablowitz and P. Clarkson, Solitons, nonlinear evolution equations and inverse scattering, London Mathematical Society Lecture Note Series, 149. Cambridge University Press, Cambridge, 1991.

[2] M.A. Alejo, Geometric Breathers of the $m K d V$ Equation, Acta Appl. Math. 121, no. 1 (2012), p.137-155.

[3] M.A. Alejo, C. Gorria and L. Vega, Discrete conservation laws and the convergence of long time simulations of the $m K d V$ equation, J. Comp. Phys. 235 (2013), 274-285.

[4] M.A. Alejo and C. Muñoz, Nonlinear stability of $m K d V$ breathers, to appear in Comm. Math. Phys. arXiv:1206.3157 
[5] M.A. Alejo, C. Muñoz, and L. Vega, The Gardner equation and the $L^{2}$-stability of the $N$ soliton solution of the Korteweg-de Vries equation, Transactions of the AMS, 365 no. 1 (2013), 195-212.

[6] S. Aubry, Breathers in nonlinear lattices: Existence, linear stability and quantization, Physica D no. 103 (1997), 201-250.

[7] T.B. Benjamin, The stability of solitary waves, Proc. Roy. Soc. London A 328, (1972) 153183.

[8] B. Birnir, H.P. McKean, and A. Weinstein, The rigidity of sine-Gordon breathers, Comm. Pure Appl. Math. 47, 1043-1051 (1994).

[9] J.L. Bona, P. Souganidis, and W. Strauss, Stability and instability of solitary waves of Korteweg-de Vries type, Proc. Roy. Soc. London 411 (1987), 395-412.

[10] J.L. Bona, S. Vento and F. B. Weissler, Singularity formation and blowup of complex-valued solutions of the modified KdV-equation, preprint arXiv: $1201.0442 \mathrm{v} 1$.

[11] T. Buckmaster and H. Koch, The Korteweg-de Vries equation at $H^{-1}$ regularity, preprint arXiv:1112.4657.

[12] M. Christ, J. Holmer, and D. Tataru, Low regularity bounds for $m K d V$, arXiv:1207.6738

[13] J. Colliander, M. Keel, G. Staffilani, H. Takaoka, and T. Tao, Sharp global well-posedness for $K d V$ and modified $K d V$ on $\mathbb{R}$ and $\mathbb{T}$, J. Amer. Math. Soc. 16 (2003), no. 3, 705-749 (electronic).

[14] J. Denzler, Nonpersistence of breather families for the perturbed Sine-Gordon equation, Commun. Math. Phys. 158, 397-430 (1993).

[15] Deift, P. and Xin Zhou, A steepest descent method for oscillatory Riemann-Hilbert problems. Asymptotics for the MKdV equation, Ann. of Math. (2) 137 (1993), 295-368.

[16] N. Ercolani, M. G. Forest and D. W. McLauglin, Modulational stability of two-phase sineGordon wave trains, Studies in Applied Math (2), 91-101 (1985).

[17] C.S. Gardner, M.D. Kruskal, and R. Miura, Korteweg-de Vries equation and generalizations. II. Existence of conservation laws and constants of motion, J. Math. Phys. 9, no. 8 (1968), 1204-1209.

[18] M. Grillakis, J. Shatah, and W. Strauss, Stability theory of solitary waves in the presence of symmetry. I. J. Funct. Anal. 74 (1987), no. 1, 160-197.

[19] D. B. Henry, J.F. Perez and W. F. Wreszinski, Stability Theory for Solitary-Wave Solutions of Scalar Field Equations, Comm. Math. Phys. 85, 351-361 (1982).

[20] R. Hirota, Exact solution of the modified Korteweg-de Vries equation for multiple collisions of solitons, J. Phys. Soc. Japan, 33, no. 5 (1972) 1456-1458.

[21] A. Hoffman, and C.E. Wayne, Orbital stability of localized structures via Bäcklund transfomations, preprint to appear in DIE.

[22] S. Kwon, and T. Oh, On unconditional well-posedness of modified $K d V$, to appear in IMRN. arXiv:1007.0270v2.

[23] C.E. Kenig, G. Ponce, and L. Vega, Well-posedness and scattering results for the generalized Korteweg-de Vries equation via the contraction principle, Comm. Pure Appl. Math. 46, (1993) 527-620.

[24] C.E. Kenig, G. Ponce, and L. Vega, On the ill-posedness of some canonical dispersive equations, Duke Math. J. 106 (2001), no. 3, 617-633.

[25] G.L. Lamb, Elements of Soliton Theory, Pure Appl. Math., Wiley, New York, 1980.

[26] P.D. Lax, Integrals of nonlinear equations of evolution and solitary waves, Comm. Pure Appl. Math. 21, (1968) 467-490.

[27] J.H. Maddocks and R.L. Sachs, On the stability of KdV multi-solitons, Comm. Pure Appl. Math. 46, 867-901 (1993).

[28] T. Mizumachi and D. Pelinovsky, Bäcklund transformation and $L^{2}$-stability of NLS solitons, to appear in IMRN, arXiv:1011.5922 2 .

[29] Y. Martel and F. Merle, Description of two soliton collision for the quartic gKdV equations, to appear in Annals of Mathematics.

[30] Y. Martel, and F. Merle, Blow up in finite time and dynamics of blow up solutions for the critical generalized KdV equation, J. Amer. Math. Soc. 15 (2002), 617-664.

[31] Y. Martel and F. Merle, Asymptotic stability of solitons of the subcritical gKdV equations revisited, Nonlinearity 18 (2005) 55-80. 
[32] Y. Martel, F. Merle, and T.P. Tsai, Stability and asymptotic stability in the energy space of the sum of $N$ solitons for subcritical gKdV equations, Comm. Math. Phys. 231 (2002) 347-373.

[33] F. Merle, On Uniqueness and Continuation Properties after Blow-up Time of Self-similar Solutions of Nonlinear Schrödinger Equation with Critical Exponent and Critical Mass, Comm. Pure Applied Math. Vol. XLV, 203-254 (1992).

[34] F. Merle and L. Vega, $L^{2}$ stability of solitons for KdV equation, Int. Math. Res. Not. 2003, no. $13,735-753$.

[35] R.L. Pego and M.I. Weinstein, Asymptotic stability of solitary waves, Commun. Math. Phys. 164, 305-349 (1994).

[36] P.C. Schuur, Asymptotic analysis of soliton problems. An inverse scattering approach, Lecture Notes in Mathematics, 1232. Springer-Verlag, Berlin, 1986. viii+180 pp.

[37] A. Soffer and M.I. Weinstein, Resonances, radiation damping and instability in Hamiltonian nonlinear wave equations, Invent. Math. 136 (1999), no. 1, 9-74.

[38] M. Wadati, The modified Korteweg-de Vries Equation, J. Phys. Soc. Japan, 34, no.5, (1973), $1289-1296$.

[39] H. D. Wahlquist and F. B. Estabrook, Bäcklund transformation for solutions of the Kortewegde Vries equation, Phys. Rev. Lett. 31 no. 23 (1973), 1386-1390.

[40] M.I. Weinstein, Lyapunov stability of ground states of nonlinear dispersive evolution equations, Comm. Pure Appl. Math. 39, (1986) 51-68.

impa(Instituto Nacional de Matemática Pura e Aplicada), Rio de Janeiro, Brasil

E-mail address: malejo@impa.br

CNRS and Laboratoire de Mathématiques D'Orsay UMR 8628, BÂt. 425 Faculté des Sciences d’Orsay, Université Paris-Sud F-91405 Orsay Cedex France

E-mail address: claudio.munoz@math.u-psud.fr 